\title{
Properties of the zero-and-one inflated Poisson distribution and likelihood-based inference methods
}

\author{
Chi Zhang, Guo-Liang Tian*, And Kai-Wang NG
}

To model count data with excess zeros and excess ones, in their unpublished manuscript, Melkersson and Olsson (1999) extended the zero-inflated Poisson distribution to a zero-and-one-inflated Poisson (ZOIP) distribution. However, the distributional theory and corresponding properties of the ZOIP have not yet been explored, and likelihoodbased inference methods for parameters of interest were not well developed. In this paper, we extensively study the ZOIP distribution by first constructing five equivalent stochastic representations for the ZOIP random variable and then deriving other important distributional properties. Maximum likelihood estimates of parameters are obtained by both the Fisher scoring and expectation-maximization algorithms. Bootstrap confidence intervals for parameters of interest and testing hypotheses under large sample sizes are provided. Simulations studies are performed and five real data sets are used to illustrate the proposed methods.

KEYWORDS AND PHRASES: EM algorithm, Fisher scoring algorithm, One-inflated Poisson distribution, Zero-inflated Poisson model, Zero-and-one inflated Poisson model.

\section{INTRODUCTION}

Poisson model provides a standard and popular framework to model count data. The over-dispersion problem emerges in modeling count data due to the greater incidence of zeros than that permitted by the traditional Poisson distribution. Lambert [17] proposed the zero-inflated Poisson (ZIP) regression model to handle count data with excess zeros. Some examples of ZIP distribution can be found in Neyman [22], Cohen [4], Singh [29], Martin and Katti [19], Kemp [16], and Mullahy [21]. Gupta et al. [11] developed a zero-adjusted generalized Poisson distribution including the ZIP distribution as a special case. Subsequently, Ridout et al. [24] reviewed some useful models for fitting count data with excess zeros. Carrivick et al. [3] applied the ZIP model to evaluate the effectiveness of a consultative manual handling workplace risk assessment team in reducing the risk of occupational injury among cleaners within a hospital.

\footnotetext{
*Corresponding author.
}

The over-dispersion may result from other phenomena such as a high proportion of both zeros and ones in the count data. For example, Eriksson and Åberg [8] reported a two-year panel data from Swedish Level of Living Surveys in 1974 and 1991, where the visits to a dentist have higher proportions of zeros and ones and one-visit observations are even much more frequent than zero-visits. It is common to visit a dentist for a routine control, e.g., school-children go to a dentist once a year almost as a rule. As the second example, Carrivick et al. [3] reported that much of the data collected on occupational safety involves accident or injury counts with many zeros and ones. In general, the safety regulations protect most workers from injury so that many zeros are observed. However, no perfect protection exists, such that there must be somebody suffering an accident or injury. This experience would be a signal to warn other workers to be much more cautious and to avoid more accidents. Hence there will be respectively more one observed indicating the majority of people having only one accident or none than those with two or more injuries.

Thus, the ZIP is no longer an appropriate distribution to model such count data with extra zeros and extra ones. As an extension of the ZIP, a so-called zero-and-one-inflated Poisson (ZOIP) distribution proposed by Melkersson and Olsson [20] is then a useful tool to capture the characteristics of such count data. The major goal of Melkersson and Olsson [20] is to fit the dentist visiting data with covariates in Sweden. Later, Saito and Rodrigues [25] presented a Bayesian analysis of the same dentist visiting data without considering any covariates by the data augmentation algorithm (Tanner and Wong, [30]). However, the distributional theory and corresponding properties of the ZOIP have not yet been explored, and likelihood-based methods for parameters of interest were not well developed, because, to our best knowledge, only two papers (i.e., Melkersson and Olsson, [20]; Saito and Rodrigues, [25]) involve the ZOIP to date. The main objective of this paper is to fill the gap.

For convenience, in this paper we denote a degenerate distribution at a single point $c$ by $\xi \sim \operatorname{Degenerate}(c)$, where $\xi$ is a random variable with probability mass function (pmf) $\operatorname{Pr}(\xi=c)=1$ and $c$ is a constant.

Let $\xi_{0} \sim$ Degenerate(0), $\xi_{1} \sim$ Degenerate(1), $X \sim$ $\operatorname{Poisson}(\lambda)$ and they are independent. A discrete random 
variable $Y$ is said to follow a ZOIP distribution, denoted by $Y \sim \operatorname{ZOIP}\left(\phi_{0}, \phi_{1} ; \lambda\right)$, if its pmf is (Melkersson and Olsson, [20])

$$
\text { (1.1) } \begin{aligned}
& f\left(y \mid \phi_{0}, \phi_{1} ; \lambda\right) \\
= & \phi_{0} \operatorname{Pr}\left(\xi_{0}=y\right)+\phi_{1} \operatorname{Pr}\left(\xi_{1}=y\right)+\phi_{2} \operatorname{Pr}(X=y) \\
= & \left\{\begin{array}{ll}
\phi_{0}+\phi_{2} \mathrm{e}^{-\lambda}, \quad \text { if } y=0, \\
\phi_{1}+\phi_{2} \lambda \mathrm{e}^{-\lambda}, & \text { if } y=1, \\
\phi_{2} \frac{\lambda^{y} \mathrm{e}^{-\lambda}}{y !}, & \text { if } y=2,3, \ldots
\end{array}=\right. \\
= & \left(\phi_{0}+\phi_{2} \mathrm{e}^{-\lambda}\right) I(y=0)+\left(\phi_{1}+\phi_{2} \lambda \mathrm{e}^{-\lambda}\right) I(y=1) \\
+ & \left(\phi_{2} \frac{\lambda^{y} \mathrm{e}^{-\lambda}}{y !}\right) I(y \geqslant 2),
\end{aligned}
$$

where $\phi_{0} \in[0,1)$ and $\phi_{1} \in[0,1)$ respectively denote the unknown proportions for incorporating extra-zeros and extraones than those allowed by the traditional Poisson distribution, and $\phi_{2} \hat{=} 1-\phi_{0}-\phi_{1} \in(0,1]$. The ZOIP distribution is a mixture of two degenerate distributions with all mass at zero and one, respectively and a Poisson $(\lambda)$ distribution. In particular, when $\phi_{0}=0$, the ZOIP distribution reduces to one-inflated Poisson (OIP) distribution (denoted by $\left.\operatorname{OIP}\left(\phi_{1}, \lambda\right)\right)$; when $\phi_{1}=0$, the ZOIP distribution reduces to ZIP distribution (denoted by $\operatorname{ZIP}\left(\phi_{0}, \lambda\right)$ ); when $\phi_{0}=\phi_{1}=0$, the ZOIP distribution becomes the traditional Poisson distribution.

The remainder of the paper is organized as follows. Section 2 presents five equivalent stochastic representations for the ZOIP random variable. Section 3 develops other distributional properties. In Section 4, we derive the Fisher scoring algorithm and the expectation-maximization (EM) algorithm for finding the MLEs of parameters. Bootstrap confidence intervals are also provided. The likelihood ratio test and the score test for one inflation, zero inflation and simultaneous zero-and-one inflation are developed in Section 5. Simulation studies to compare the likelihood ratio test with the score test are conducted in Section 6. In Section 7, five real data sets are used to illustrate the proposed methods. A discussion is given in Section 8 .

\section{VARIOUS STOCHASTIC REPRESENTATIONS AND THEIR EQUIVALENCE}

In this section, we present five different stochastic representation (SR) for the random variable $Y \sim$ $\operatorname{ZOIP}\left(\phi_{0}, \phi_{1} ; \lambda\right)$ and show their equivalence. These SRs reveal the relationship of the $\operatorname{ZOIP}\left(\phi_{0}, \phi_{1} ; \lambda\right)$ distribution with Degenerate(0), Degenerate(1), Poisson $(\lambda)$, $\operatorname{Bernoulli}\left(\phi_{1} /\left(\phi_{0}+\phi_{1}\right)\right), \operatorname{ZIP}\left(\phi_{0} /\left(1-\phi_{1}\right), \lambda\right), \operatorname{ZTP}(\lambda)$ and $\operatorname{OTP}(\lambda)$.

\subsection{The first stochastic representation}

Let $\mathbf{z}=\left(Z_{0}, Z_{1}, Z_{2}\right)^{\top} \sim$ Multinomial $\left(1 ; \phi_{0}, \phi_{1}, \phi_{2}\right), X \sim$ $\operatorname{Poisson}(\lambda)$, and $\mathbf{z}$ and $X$ be mutually independent (denoted by $\mathbf{z} \Perp X)$. It is easy to show that the first SR of the random variable $Y \sim \operatorname{ZOIP}\left(\phi_{0}, \phi_{1} ; \lambda\right)$ is given by

$$
\begin{aligned}
Y & \stackrel{d}{=} Z_{0} \cdot 0+Z_{1} \cdot 1+Z_{2} X=Z_{1}+Z_{2} X \\
& = \begin{cases}0, & \text { with probability } \phi_{0}, \\
1, & \text { with probability } \phi_{1}, \\
X, & \text { with probability } \phi_{2},\end{cases}
\end{aligned}
$$

where the symbol "d $=$ " means that the random variables in both sides of the equality have the same distribution. The justification of this fact is as follows. By noting that $Z_{0}+Z_{1}+Z_{2}=1$ and $\operatorname{Pr}\left(Z_{i}=1\right)=\phi_{i}$ for $i=0,1,2$, from (2.1), we have

$$
\left\{\begin{aligned}
\operatorname{Pr}(Y=0) & =\operatorname{Pr}\left(Z_{0}=1\right)+\operatorname{Pr}\left(Z_{2}=1, X=0\right) \\
& =\phi_{0}+\phi_{2} \mathrm{e}^{-\lambda} \\
\operatorname{Pr}(Y=1) & =\operatorname{Pr}\left(Z_{1}=1\right)+\operatorname{Pr}\left(Z_{2}=1, X=1\right) \\
& =\phi_{1}+\phi_{2} \lambda \mathrm{e}^{-\lambda} \\
\operatorname{Pr}(Y=y) & =\operatorname{Pr}\left(Z_{2}=1, X=y\right)=\phi_{2} \frac{\lambda^{y} \mathrm{e}^{-\lambda}}{y !}, y \geqslant 2
\end{aligned}\right.
$$

which is identical to (1.1), implying that $Y \sim$ $\operatorname{ZOIP}\left(\phi_{0}, \phi_{1} ; \lambda\right)$. The SR (2.1) means that $Y \sim$ $\operatorname{ZOIP}\left(\phi_{0}, \phi_{1} ; \lambda\right)$ is a mixture of three distributions: Degenerate(0), Degenerate(1) and Poisson $(\lambda)$.

\subsection{The second stochastic representation}

Alternatively, we can develop the second SR of the zero-and-one inflated Poisson random variable. Let $Z \sim$ $\operatorname{Bernoulli}(1-\phi), \eta \sim \operatorname{Bernoulli}(p), X \sim \operatorname{Poisson}(\lambda)$ and $(Z, \eta, X)$ be mutually independent. Then

$$
Y \stackrel{d}{=}(1-Z) \eta+Z X= \begin{cases}\eta, & \text { with probability } \phi, \\ X, & \text { with probability } 1-\phi\end{cases}
$$

follows the distribution $\operatorname{ZOIP}\left(\phi_{0}, \phi_{1} ; \lambda\right)$ with $\phi_{0}=\phi(1-p)$ and $\phi_{1}=\phi p$. This fact can be verified as follows:

$$
\left\{\begin{aligned}
\operatorname{Pr}(Y=0) & =\operatorname{Pr}(Z=0, Y=0)+\operatorname{Pr}(Z=1, Y=0) \\
& =\operatorname{Pr}(Z=0, \eta=0)+\operatorname{Pr}(Z=1, X=0) \\
& =\phi(1-p)+(1-\phi) \mathrm{e}^{-\lambda} \\
\operatorname{Pr}(Y=1) & =\operatorname{Pr}(Z=0, Y=1)+\operatorname{Pr}(Z=1, Y=1) \\
& =\operatorname{Pr}(Z=0, \eta=1)+\operatorname{Pr}(Z=1, X=1) \\
& =\phi p+(1-\phi) \lambda \mathrm{e}^{-\lambda}, \\
\operatorname{Pr}(Y=y) & =\operatorname{Pr}(Z=1, X=y) \\
& =(1-\phi) \lambda^{y} \mathrm{e}^{-\lambda} / y !, \quad y \geqslant 2 .
\end{aligned}\right.
$$


By comparing (2.4) with (2.2), we obtain a one-to-one mapping between $(\phi, p)$ and $\left(\phi_{0}, \phi_{1}\right)$ :

$$
\left\{\begin{array} { l l } 
{ \phi ( 1 - p ) } & { = \phi _ { 0 } , } \\
{ \phi p } & { = \phi _ { 1 } , } \\
{ 1 - \phi } & { = \phi _ { 2 } , }
\end{array} \Longleftrightarrow \left\{\begin{array}{l}
\phi=\phi_{0}+\phi_{1}, \\
p=\frac{\phi_{1}}{\phi_{0}+\phi_{1}} .
\end{array}\right.\right.
$$

Now let $\mathbf{z}^{*}=\left(Z_{0}^{*}, Z_{1}^{*}, Z_{2}^{*}\right)^{\top} \sim \operatorname{Multinomial}\left(1 ; \phi_{0}^{*}, \phi_{1}^{*}, \phi_{2}^{*}\right)$, $V_{0} \sim \operatorname{ZTP}(\lambda)$ and $\mathbf{z}^{*} \Perp V_{0}$. Then

The SR (2.3) indicates that $Y \sim \operatorname{ZOIP}\left(\phi_{0}, \phi_{1} ; \lambda\right)$ is also a mixture of a Bernoulli $\left(\phi_{1} /\left(\phi_{0}+\phi_{1}\right)\right)$ distribution and a $\operatorname{Poisson}(\lambda)$ distribution.

\subsection{The third stochastic representation}

Now we consider the third SR of $Y \sim \operatorname{ZOIP}\left(\phi_{0}, \phi_{1} ; \lambda\right)$. Let $Z \sim \operatorname{Bernoulli}(1-\phi), \xi_{1} \sim \operatorname{Degenerate}(1), Y^{*} \sim$ $\operatorname{ZIP}\left(\phi^{*}, \lambda\right)$ and $\left(Z, \xi_{1}, Y^{*}\right)$ be mutually independent. Then $(2.6)$

$$
Y \stackrel{d}{=}(1-Z) \xi_{1}+Z Y^{*}= \begin{cases}1, & \text { with probability } \phi \\ Y^{*}, & \text { with probability } 1-\phi\end{cases}
$$

follows the distribution $\operatorname{ZOIP}\left(\phi_{0}, \phi_{1} ; \lambda\right)$ with $\phi_{0}=(1-\phi) \phi^{*}$ and $\phi_{1}=\phi$. This fact can be shown as follows:

$$
\left\{\begin{aligned}
\operatorname{Pr}(Y=0) & =\operatorname{Pr}\left(Z=1, Y^{*}=0\right) \\
& =(1-\phi)\left[\phi^{*}+\left(1-\phi^{*}\right) \mathrm{e}^{-\lambda}\right], \\
\operatorname{Pr}(Y=1) & =\operatorname{Pr}(Z=0)+\operatorname{Pr}\left(Z=1, Y^{*}=1\right) \\
& =\phi+(1-\phi)\left(1-\phi^{*}\right) \lambda \mathrm{e}^{-\lambda}, \\
\operatorname{Pr}(Y=y) & =\operatorname{Pr}\left(Z=1, Y^{*}=y\right) \\
& =(1-\phi)\left(1-\phi^{*}\right) \lambda^{y} \mathrm{e}^{-\lambda} / y !, \quad y \geqslant 2 .
\end{aligned}\right.
$$

By comparing (2.7) with (2.2), we obtain a one-to-one mapping between $\left(\phi, \phi^{*}\right)$ and $\left(\phi_{0}, \phi_{1}\right)$ :

$$
\begin{aligned}
\left\{\begin{array}{lll}
(1-\phi) \phi^{*} & =\phi_{0}, \\
\phi & = & \phi_{1}, \\
(1-\phi)\left(1-\phi^{*}\right) & = & \phi_{2},
\end{array}\right. \\
\Longleftrightarrow\left\{\begin{array}{l}
\phi=\phi_{1}, \\
\phi^{*}=\frac{\phi_{0}}{1-\phi_{1}} .
\end{array}\right.
\end{aligned}
$$

The SR (2.6) shows that $Y \sim \operatorname{ZOIP}\left(\phi_{0}, \phi_{1} ; \lambda\right)$ is also a mixture of a degenerate distribution with all mass at 1 and a $\operatorname{ZIP}\left(\phi^{*}, \lambda\right)$ distribution.

\subsection{The fourth stochastic representation}

To derive the fourth SR of $Y \sim \operatorname{ZOIP}\left(\phi_{0}, \phi_{1} ; \lambda\right)$, we first introduce the zero-truncated Poisson (ZTP) distribution (David and Johnson, [6]), denoted by $V_{0} \sim \operatorname{ZTP}(\lambda)$, whose pmf is defined as

$$
\operatorname{Pr}\left(V_{0}=v\right)=\frac{\lambda^{v} \mathrm{e}^{-\lambda} / v !}{1-\mathrm{e}^{-\lambda}}, \quad v=1,2, \ldots, \infty .
$$

$$
Y \stackrel{d}{=} Z_{1}^{*}+Z_{2}^{*} V_{0}= \begin{cases}0, & \text { with probability } \phi_{0}^{*} \\ 1, & \text { with probability } \phi_{1}^{*} \\ V_{0}, & \text { with probability } \phi_{2}^{*}\end{cases}
$$

follows the distribution $\operatorname{ZOIP}\left(\phi_{0}, \phi_{1} ; \lambda\right)$ with $\phi_{0}=\phi_{0}^{*}-$ $\phi_{2}^{*} /\left(\mathrm{e}^{\lambda}-1\right)$ and $\phi_{1}=\phi_{1}^{*}$. This fact can be verified as follows: $(2.11)$

$$
\left\{\begin{aligned}
\operatorname{Pr}(Y=0) & =\operatorname{Pr}\left(Z_{0}^{*}=1\right)=\phi_{0}^{*} \\
\operatorname{Pr}(Y=1) & =\operatorname{Pr}\left(Z_{1}^{*}=1\right)+\operatorname{Pr}\left(Z_{2}^{*}=1, V_{0}=1\right) \\
& =\phi_{1}^{*}+\phi_{2}^{*} \lambda \mathrm{e}^{-\lambda} /\left(1-\mathrm{e}^{-\lambda}\right), \\
\operatorname{Pr}(Y=y) & =\operatorname{Pr}\left(Z_{2}^{*}=1, V_{0}=y\right) \\
& =\phi_{2}^{*} \lambda^{y} \mathrm{e}^{-\lambda} /\left[y !\left(1-\mathrm{e}^{-\lambda}\right)\right], \quad y \geqslant 2 .
\end{aligned}\right.
$$

By comparing (2.11) with (2.2), we obtain a one-to-one mapping between $\left(\phi_{0}^{*}, \phi_{1}^{*}, \phi_{2}^{*}\right)$ and $\left(\phi_{0}, \phi_{1}, \phi_{2}\right)$ :

$$
\begin{array}{r}
\left\{\begin{array}{l}
\phi_{0}^{*}=\phi_{0}+\phi_{2} \mathrm{e}^{-\lambda}, \\
\phi_{1}^{*}=\phi_{1}, \\
\phi_{2}^{*}=\phi_{2}\left(1-\mathrm{e}^{-\lambda}\right),
\end{array}\right. \\
\Longleftrightarrow \begin{cases}\phi_{0} & =\phi_{0}^{*}-\frac{\phi_{2}^{*}}{\mathrm{e}^{\lambda}-1}, \\
\phi_{1} & =\phi_{1}^{*}, \\
\phi_{2} & =\phi_{2}^{*} \mathrm{e}^{\lambda} /\left(\mathrm{e}^{\lambda}-1\right) .\end{cases}
\end{array}
$$

The SR (2.10) shows that $Y \sim \operatorname{ZOIP}\left(\phi_{0}, \phi_{1} ; \lambda\right)$ is also a mixture of three distributions: Degenerate(0), Degenerate(1) and $\operatorname{ZTP}(\lambda)$.

\subsection{The fifth stochastic representation}

First of all, we introduce the one-truncated Poisson (OTP) distribution (Cohen, [5]; Hilbe, [13], p. 388) with pmf defined by

$$
\operatorname{Pr}\left(V_{1}=v\right)=\frac{\lambda^{v} \mathrm{e}^{-\lambda} / v !}{1-\mathrm{e}^{-\lambda}-\lambda \mathrm{e}^{-\lambda}}, \quad v=2,3, \ldots, \infty,
$$

and denote it by $V_{1} \sim \operatorname{OTP}(\lambda)$. Now let $\mathbf{z}^{*}=$ $\left(Z_{0}^{*}, Z_{1}^{*}, Z_{2}^{*}\right)^{\top} \sim \operatorname{Multinomial}\left(1 ; \phi_{0}^{*}, \phi_{1}^{*}, \phi_{2}^{*}\right), V_{1} \sim \operatorname{OTP}(\lambda)$ and $\mathbf{z}^{*} \Perp V_{1}$. Then

(2.14) $\quad \stackrel{\stackrel{d}{=}}{=} Z_{1}^{*}+Z_{2}^{*} V_{1}= \begin{cases}0, & \text { with probability } \phi_{0}^{*} \\ 1, & \text { with probability } \phi_{1}^{*} \\ V_{1}, & \text { with probability } \phi_{2}^{*}\end{cases}$

follows the distribution $\operatorname{ZOIP}\left(\phi_{0}, \phi_{1} ; \lambda\right)$ with $\phi_{0}=\phi_{0}^{*}-$ $\phi_{2}^{*} /\left(\mathrm{e}^{\lambda}-1-\lambda\right)$ and $\phi_{1}=\phi_{1}^{*}-\phi_{2}^{*} \lambda /\left(\mathrm{e}^{\lambda}-1-\lambda\right)$. This fact 
can be verified as follows:

(2.15)

$$
\left\{\begin{aligned}
\operatorname{Pr}(Y=0) & =\operatorname{Pr}\left(Z_{0}^{*}=1\right)=\phi_{0}^{*}, \\
\operatorname{Pr}(Y=1) & =\operatorname{Pr}\left(Z_{1}^{*}=1\right)=\phi_{1}^{*}, \\
\operatorname{Pr}(Y=y) & =\operatorname{Pr}\left(Z_{2}^{*}=1, V_{1}=y\right) \\
& =\phi_{2}^{*} \lambda^{y} \mathrm{e}^{-\lambda} /\left[y !\left(1-\mathrm{e}^{-\lambda}-\lambda \mathrm{e}^{-\lambda}\right)\right], y \geqslant 2 .
\end{aligned}\right.
$$

By comparing (2.15) with (2.2), we obtain a one-to-one mapping between $\left(\phi_{0}^{*}, \phi_{1}^{*}, \phi_{2}^{*}\right)$ and $\left(\phi_{0}, \phi_{1}, \phi_{2}\right)$ :

$$
\begin{aligned}
& \left\{\begin{array}{l}
\phi_{0}^{*}=\phi_{0}+\phi_{2} \mathrm{e}^{-\lambda}, \\
\phi_{1}^{*}=\phi_{1}+\phi_{2} \lambda \mathrm{e}^{-\lambda}, \\
\phi_{2}^{*}=\phi_{2}\left(1-\mathrm{e}^{-\lambda}-\lambda \mathrm{e}^{-\lambda}\right),
\end{array}\right. \\
& \Longleftrightarrow\left\{\begin{array}{l}
\phi_{0}=\phi_{0}^{*}-\phi_{2}^{*} /\left(\mathrm{e}^{\lambda}-1-\lambda\right), \\
\phi_{1}=\phi_{1}^{*}-\phi_{2}^{*} \lambda /\left(\mathrm{e}^{\lambda}-1-\lambda\right), \\
\phi_{2}=\phi_{2}^{*} \mathrm{e}^{\lambda} /\left(\mathrm{e}^{\lambda}-1-\lambda\right) .
\end{array}\right.
\end{aligned}
$$

The SR (2.14) shows that $Y \sim \operatorname{ZOIP}\left(\phi_{0}, \phi_{1} ; \lambda\right)$ is also a mixture of three distributions: Degenerate $(0)$, Degenerate $(1)$ and $\operatorname{OTP}(\lambda)$.

\section{DISTRIBUTIONAL PROPERTIES}

\subsection{The cumulative distribution function}

Let $Y \sim \operatorname{ZOIP}\left(\phi_{0}, \phi_{1} ; \lambda\right)$. For any non-negative real number $y$, the cumulative distribution function of $Y$ is given by

$$
\begin{array}{ll} 
& \operatorname{Pr}(Y \leqslant y) \\
= & \left(\phi_{0}+\phi_{2} \mathrm{e}^{-\lambda}\right) I(0 \leqslant y<1) \\
& +\left(\phi_{0}+\phi_{1}+\phi_{2} \sum_{i=0}^{\lfloor y\rfloor} \frac{\lambda^{i} \mathrm{e}^{-\lambda}}{i !}\right) I(y \geqslant 1) \\
=\quad & \left(\phi_{0}+\phi_{2} \mathrm{e}^{-\lambda}\right) I(0 \leqslant y<1) \\
& +\left[\phi_{0}+\phi_{1}+\phi_{2} \frac{\Gamma(\lfloor y+1\rfloor, \lambda)}{\lfloor y\rfloor !}\right] I(y \geqslant 1),
\end{array}
$$

where $\lfloor k\rfloor$ denotes the largest integer not greater than $k$, and

$$
\Gamma(k, \lambda) \hat{=} \int_{\lambda}^{\infty} t^{k-1} \mathrm{e}^{-t} \mathrm{~d} t
$$

is the upper incomplete gamma function.

\subsection{Moments}

The SR (2.1) is a useful tool to derive the moments of the ZOIP random variable. Since $\left(Z_{0}, Z_{1}, Z_{2}\right)^{\top} \sim$ Multinomial $\left(1 ; \phi_{0}, \phi_{1}, \phi_{2}\right)$, we have $E\left(Z_{i}\right)=\phi_{i}, \operatorname{Var}\left(Z_{i}\right)=$ $\phi_{i}\left(1-\phi_{i}\right), \operatorname{Cov}\left(Z_{i}, Z_{j}\right)=-\phi_{i} \phi_{j}$ and $E\left(Z_{i} Z_{j}\right)=0$ for $i \neq j$. From (2.1), we immediately obtain

$$
E(Y)=\phi_{1}+\phi_{2} \lambda \hat{=} \mu
$$

$$
\begin{aligned}
E\left(Y^{2}\right) & =\phi_{1}+\phi_{2}\left(\lambda+\lambda^{2}\right), \\
\operatorname{Var}(Y) & =\mu-\mu^{2}+\left(\mu-\phi_{1}\right)^{2} / \phi_{2} .
\end{aligned}
$$

In general, for any positive integers $r$ and $s$, we have $Z_{i}^{r} Z_{j}^{s} \sim$ Degenerate(0) for $i \neq j$. Let $n$ be an arbitrary positive integer, we have

$$
\begin{aligned}
E\left(Y^{n}\right) & =\sum_{k=0}^{n}\left(\begin{array}{l}
n \\
k
\end{array}\right) E\left(Z_{1}^{k} Z_{2}^{n-k}\right) E\left(X^{n-k}\right) \\
& =\phi_{1}+\phi_{2} E\left(X^{n}\right) .
\end{aligned}
$$

Alternatively, the SR (2.3) can be applied to calculate $E\left(Y^{n}\right)$, and we can obtain the same result as (3.4) by noting that $(1-Z)^{r} Z^{s} \sim$ Degenerate(0) for any Bernoulli random variable $Z$, where $r$ and $s$ are two arbitrary positive integers.

\subsection{Moment generating function}

By using the formula of $E\left(W_{1}\right)=E\left[E\left(W_{1} \mid W_{2}\right)\right]$, the moment generating function of $Y \sim \operatorname{ZOIP}\left(\phi_{0}, \phi_{1} ; \lambda\right)$ is given by

$$
\begin{array}{ll} 
& M_{Y}(t)=E[\exp (t Y)] \\
\stackrel{(2.3)}{=} & E\{\exp [t(1-Z) \eta+t Z X]\} \\
= & E\{E\{\exp [t(1-Z) \eta+t Z X] \mid Z\}\} \\
= & E\left[M_{\eta}(t(1-Z)) \cdot M_{X}(t Z)\right] \\
= & E\left\{\left[p \mathrm{e}^{t(1-Z)}+1-p\right] \cdot \exp \left[\lambda\left(\mathrm{e}^{t Z}-1\right)\right]\right\} \\
= & \phi\left(p \mathrm{e}^{t}+1-p\right)+(1-\phi) \exp \left[\lambda\left(\mathrm{e}^{t}-1\right)\right] .
\end{array}
$$

\subsection{Conditional distributions based on the first SR}

In Section 2.1, we assumed that $\mathbf{z}=\left(Z_{0}, Z_{1}, Z_{2}\right)^{\top} \sim$ Multinomial $\left(1 ; \phi_{0}, \phi_{1}, \phi_{2}\right)$ and $Y \sim \operatorname{ZOIP}\left(\phi_{0}, \phi_{1} ; \lambda\right)$. It is clear that $\mathbf{z}$ only takes one of the three base-vectors $(1,0,0)^{\top}$, $(0,1,0)^{\top}$ and $(0,0,1)^{\top}$. We first consider the joint conditional distribution of $\mathbf{z} \mid Y$, which is stated as follows.

Theorem 1. (Joint conditional distribution of $\mathbf{z} \mid Y$ ). Let $\mathbf{z} \sim$ Multinomial $\left(1 ; \phi_{0}, \phi_{1}, \phi_{2}\right)$ and $Y \sim \operatorname{ZOIP}\left(\phi_{0}, \phi_{1} ; \lambda\right)$. Based on the SR (2.1), we have

$$
\mathbf{z} \mid(Y=y) \sim \begin{cases}\text { Multinomial }\left(1 ; \psi_{1}, 0,1-\psi_{1}\right), & \text { if } y=0 \\ \text { Multinomial }\left(1 ; 0, \psi_{2}, 1-\psi_{2}\right), & \text { if } y=1 \\ \text { Multinomial }(1 ; 0,0,1), & \text { if } y \geqslant 2,\end{cases}
$$

where

$$
\psi_{1} \hat{=} \frac{\phi_{0}}{\phi_{0}+\phi_{2} \mathrm{e}^{-\lambda}} \quad \text { and } \quad \psi_{2} \hat{=} \frac{\phi_{1}}{\phi_{1}+\phi_{2} \lambda \mathrm{e}^{-\lambda}} .
$$

Proof. The joint conditional pmf of $\mathbf{z} \mid(Y=y)$ is given by $\operatorname{Pr}(\mathbf{z}=\boldsymbol{z} \mid Y=y)=\frac{\operatorname{Pr}\left(Z_{0}=z_{0}, Z_{1}=z_{1}, Z_{2}=z_{2}, Y=y\right)}{f\left(y \mid \phi_{0}, \phi_{1} ; \lambda\right)}$. 
If $y=0$, then

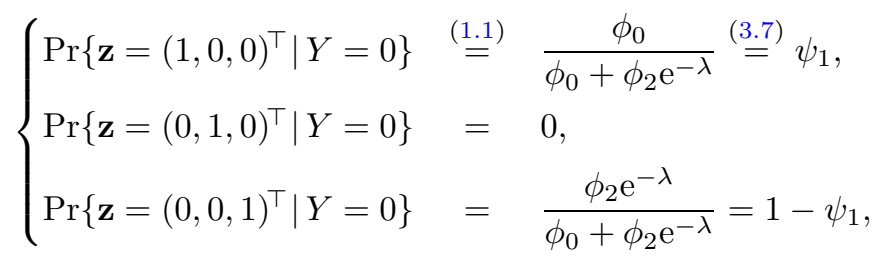

which imply the first assertion of (3.6).

If $y=1$, then

$$
\left\{\begin{array}{lll}
\operatorname{Pr}\left\{\mathbf{z}=(1,0,0)^{\top} \mid Y=1\right\} & =0, \\
\operatorname{Pr}\left\{\mathbf{z}=(0,1,0)^{\top} \mid Y=1\right\} & \stackrel{(1.1)}{=} \frac{\phi_{1}}{\phi_{1}+\phi_{2} \lambda \mathrm{e}^{-\lambda}} \\
& \stackrel{(3.7)}{=} \psi_{2}, \\
\operatorname{Pr}\left\{\mathbf{z}=(0,0,1)^{\top} \mid Y=1\right\} & =\frac{\phi_{2} \lambda \mathrm{e}^{-\lambda}}{\phi_{1}+\phi_{2} \lambda \mathrm{e}^{-\lambda}} \\
& =1-\psi_{2},
\end{array}\right.
$$

which imply the second assertion of (3.6).

If $y \geqslant 2$, then

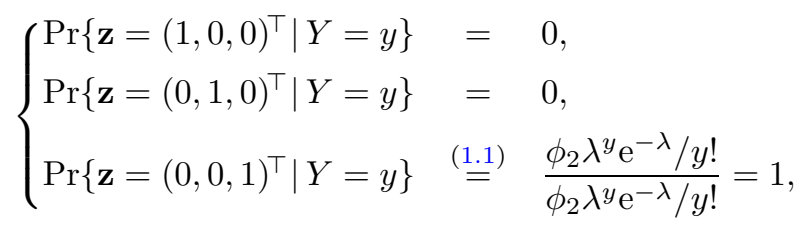

implying the last assertion of (3.6).

Next, we consider the marginal conditional distributions of $Z_{i} \mid Y$ for $i=0,1,2$. As a corollary of Theorem 1, we summarize these results as follows.

Corollary 1. (Marginal conditional distributions of $Z_{i} \mid Y$ ). Let $\mathbf{z} \sim \operatorname{Multinomial}\left(1 ; \phi_{0}, \phi_{1}, \phi_{2}\right)$ and $Y \sim \operatorname{ZOIP}\left(\phi_{0}\right.$, $\left.\phi_{1} ; \lambda\right)$. Based on the SR (2.1), we have

$$
\begin{aligned}
Z_{0} \mid(Y=y) & \sim \begin{cases}\operatorname{Bernoulli}\left(\psi_{1}\right), & \text { if } y=0, \\
\text { Degenerate }(0), & \text { if } y \neq 0,\end{cases} \\
Z_{1} \mid(Y=y) & \sim \begin{cases}\operatorname{Bernoulli}\left(\psi_{2}\right), & \text { if } y=1, \\
\text { Degenerate }(0), & \text { if } y \neq 1,\end{cases} \\
Z_{2} \mid(Y=y) & \sim \begin{cases}\text { Bernoulli }\left(1-\psi_{1}\right), & \text { if } y=0, \\
\text { Bernoulli }\left(1-\psi_{2}\right), & \text { if } y=1, \\
\text { Degenerate }(1), & \text { if } y \geqslant 2,\end{cases}
\end{aligned}
$$

where $\psi_{1}$ and $\psi_{2}$ are given by (3.7).

Finally, based on the SR (2.1), we discuss the conditional distribution of $X \mid Y$, which is stated in the following theorem.
Theorem 2. (Conditional distribution of $X \mid Y)$. Let $X \sim$ $\operatorname{Poisson}(\lambda)$ and $Y \sim \operatorname{ZOIP}\left(\phi_{0}, \phi_{1} ; \lambda\right)$. Based on the SR (2.1), we have

$$
X \mid(Y=y) \sim \begin{cases}\operatorname{ZIP}\left(1-\psi_{1}, \lambda\right), & \text { if } y=0 \\ \operatorname{OIP}\left(1-\psi_{2}, \lambda\right), & \text { if } y=1 \\ \operatorname{Degenerate}(y), & \text { if } y \geqslant 2\end{cases}
$$

where $\psi_{1}$ and $\psi_{2}$ are given by (3.7).

Proof. If $y=0$, we have

$$
\begin{array}{ll} 
& \operatorname{Pr}(X=x \mid Y=0)=\frac{\operatorname{Pr}(X=x, Y=0)}{\operatorname{Pr}(Y=0)} \\
=\quad & \frac{\operatorname{Pr}\left(X=0, Z_{1}=0\right)}{f\left(0 \mid \phi_{0}, \phi_{1} ; \lambda\right)} I(x=0) \\
& +\frac{\operatorname{Pr}\left(X=x, Z_{0}=1\right)}{f\left(0 \mid \phi_{0}, \phi_{1} ; \lambda\right)} I(x \neq 0) \\
\stackrel{(1.1)}{=} & \frac{\left(1-\phi_{1}\right) \mathrm{e}^{-\lambda}}{\phi_{0}+\phi_{2} \mathrm{e}^{-\lambda}} I(x=0)+\frac{\phi_{0} \lambda^{x} \mathrm{e}^{-\lambda} / x !}{\phi_{0}+\phi_{2} \mathrm{e}^{-\lambda}} I(x \neq 0) \\
\stackrel{(3.7)}{=} & \left(1-\psi_{1}+\psi_{1} \mathrm{e}^{-\lambda}\right) I(x=0)+\left(\psi_{1} \frac{\lambda^{x} \mathrm{e}^{-\lambda}}{x !}\right) I(x \neq 0),
\end{array}
$$

implying $X \mid(Y=0) \sim \operatorname{ZIP}\left(1-\psi_{1}, \lambda\right)$.

If $y=1$, we have

$$
\begin{array}{ll} 
& \operatorname{Pr}(X=x \mid Y=1)=\frac{\operatorname{Pr}(X=x, Y=1)}{\operatorname{Pr}(Y=1)} \\
=\quad & \frac{\operatorname{Pr}\left(X=1, Z_{0}=0\right)}{f\left(1 \mid \phi_{0}, \phi_{1} ; \lambda\right)} I(x=1) \\
& +\frac{\operatorname{Pr}\left(X=x, Z_{1}=1\right)}{f\left(1 \mid \phi_{0}, \phi_{1} ; \lambda\right)} I(x \neq 1) \\
\stackrel{(1.1)}{=} & \frac{\left(1-\phi_{0}\right) \lambda \mathrm{e}^{-\lambda}}{\phi_{1}+\phi_{2} \lambda \mathrm{e}^{-\lambda}} I(x=1)+\frac{\phi_{1} \lambda^{x} \mathrm{e}^{-\lambda} / x !}{\phi_{1}+\phi_{2} \lambda \mathrm{e}^{-\lambda}} I(x \neq 1) \\
\stackrel{(3.7)}{=} & \left(1-\psi_{2}+\psi_{2} \lambda \mathrm{e}^{-\lambda}\right) I(x=1)+\left(\psi_{2} \frac{\lambda^{x} \mathrm{e}^{-\lambda}}{x !}\right) I(x \neq 1),
\end{array}
$$

implying $X \mid(Y=1) \sim \operatorname{OIP}\left(1-\psi_{2}, \lambda\right)$.

If $y \geqslant 2$, we have

$$
\begin{aligned}
\operatorname{Pr}(X=x \mid Y=y) & =\frac{\operatorname{Pr}(X=x, Y=y)}{f\left(y \mid \phi_{0}, \phi_{1} ; \lambda\right)} \\
& \stackrel{(1.1)}{=} \frac{\operatorname{Pr}\left(X=y, Z_{2}=1\right)}{\phi_{2} \lambda^{y} \mathrm{e}^{-\lambda} / y !}=1,
\end{aligned}
$$

implying $X \mid(Y=y \geqslant 2) \sim \operatorname{Degenerate}(y)$.

\subsection{Conditional distributions based on the second SR}

In Section 2.2, we assumed that $Z \sim \operatorname{Bernoulli}(1-\phi)$, $\eta \sim \operatorname{Bernoulli}(p), X \sim \operatorname{Poisson}(\lambda)$ and $(Z, \eta, X)$ are mutually independent. According to (2.4), the pmf of $Y \sim$ $\operatorname{ZOIP}\left(\phi_{0}, \phi_{1} ; \lambda\right)$ can be rewritten as

(3.12) $f(y \mid \phi, p ; \lambda)=\left[\phi(1-p)+(1-\phi) \mathrm{e}^{-\lambda}\right] I(y=0)$ 


$$
\begin{aligned}
& +\left[\phi p+(1-\phi) \lambda \mathrm{e}^{-\lambda}\right] I(y=1) \\
& +\left[(1-\phi) \lambda^{y} \mathrm{e}^{-\lambda} / y !\right] I(y \geqslant 2) .
\end{aligned}
$$

Based on the SR (2.3), we will derive the conditional distributions of $Z|Y, \eta| Y$ and $X \mid Y$, which are given in Theorems 3,4 and 5 , respectively.

Theorem 3. (Conditional distribution of $Z \mid Y$ ). Let $Z \sim$ Bernoulli $(1-\phi)$ and the pmf of $Y$ be specified by (3.12). Based on the SR (2.3), we have

$$
Z \mid(Y=y) \sim \begin{cases}\operatorname{Bernoulli}\left(\varphi_{1}\right), & \text { if } y=0 \\ \operatorname{Bernoulli}\left(\varphi_{2}\right), & \text { if } y=1 \\ \text { Degenerate }(1), & \text { if } y \geqslant 2\end{cases}
$$

where

$$
\left\{\begin{aligned}
\varphi_{1} & \hat{=} \frac{(1-\phi) \mathrm{e}^{-\lambda}}{\phi(1-p)+(1-\phi) \mathrm{e}^{-\lambda}}=1-\psi_{1}, \\
\varphi_{2} & \hat{=} \frac{(1-\phi) \lambda \mathrm{e}^{-\lambda}}{\phi p+(1-\phi) \lambda \mathrm{e}^{-\lambda}}=1-\psi_{2},
\end{aligned}\right.
$$

and $\left(\psi_{1}, \psi_{2}\right)$ are given by $(3.7)$.

Proof. Since $Z \sim \operatorname{Bernoulli}(1-\phi), Z$ only takes the value 0 or 1. By the SR (2.3), we have

$$
\begin{aligned}
& \operatorname{Pr}(Z=1 \mid Y=y) \\
&= \frac{\operatorname{Pr}(Z=1, X=y)}{f(y \mid \phi, p ; \lambda)} \\
&= \frac{(1-\phi) \lambda^{y} \mathrm{e}^{-\lambda} / y !}{f(y \mid \phi, p ; \lambda)} \\
& \stackrel{(3.12)}{=}\left\{\begin{array}{lll}
\frac{(1-\phi) \mathrm{e}^{-\lambda}}{\phi(1-p)+(1-\phi) \mathrm{e}^{-\lambda}} & \stackrel{(3.14)}{=} \varphi_{1}, & \text { if } y=0, \\
\frac{(1-\phi) \lambda \mathrm{e}^{-\lambda}}{\phi p+(1-\phi) \lambda \mathrm{e}^{-\lambda}} & \stackrel{(3.14)}{=} \varphi_{2}, & \text { if } y=1, \\
1, & \text { if } y \geqslant 2,
\end{array}\right.
\end{aligned}
$$

$$
\begin{aligned}
& =\frac{\operatorname{Pr}(\eta=1,1-Z+Z X=y)}{f(y \mid \phi, p ; \lambda)} \\
& \stackrel{(3.12)}{=} \begin{cases}\frac{p \cdot(1-\phi) \mathrm{e}^{-\lambda}}{\phi(1-p)+(1-\phi) \mathrm{e}^{-\lambda}} & \stackrel{(3.14)}{=} p \varphi_{1}, \\
\frac{p\left[\phi+(1-\phi) \lambda \mathrm{e}^{-\lambda}\right]}{\phi p+(1-\phi) \lambda \mathrm{e}^{-\lambda}} & \stackrel{(3.14)}{=} 1-\varphi_{2}+p \varphi_{2}, \\
& \text { if } y=1, \\
\frac{p \cdot(1-\phi) \lambda^{y} \mathrm{e}^{-\lambda} / y !}{(1-\phi) \lambda^{y} \mathrm{e}^{-\lambda} / y !}, & =p \\
& \text { if } y \geqslant 2,\end{cases}
\end{aligned}
$$

which implies (3.15).

By combining Theorem 2 with (3.14), we immediately obtain the following results.

Theorem 5. (Conditional distribution of $X \mid Y)$. Let $X \sim$ Poisson $(\lambda)$ and the pmf of $Y$ be specified by (3.12). Based on the SR (2.3), we have

$$
X \mid(Y=y) \sim \begin{cases}\operatorname{ZIP}\left(\varphi_{1}, \lambda\right), & \text { if } y=0, \\ \operatorname{OIP}\left(\varphi_{2}, \lambda\right), & \text { if } y=1, \\ \operatorname{Degenerate}(y), & \text { if } y \geqslant 2,\end{cases}
$$

where $\varphi_{1}$ and $\varphi_{2}$ are given by (3.14).

\section{LIKELIHOOD-BASED INFERENCES}

Assume that $Y_{1}, \ldots, Y_{n} \stackrel{\mathrm{iid}}{\sim} \operatorname{ZOIP}\left(\phi_{0}, \phi_{1} ; \lambda\right)$ and $y_{1}, \ldots, y_{n}$ denote their realizations. Let $Y_{\mathrm{obs}}=\left\{y_{i}\right\}_{i=1}^{n}$ denote the observed data. Moreover, let $\mathbb{I}_{0} \hat{=}\left\{i \mid y_{i}=0,1 \leqslant i \leqslant n\right\}$ and $m_{0}=\sum_{i=1}^{n} I\left(y_{i}=0\right)$ denote the number of elements in $\mathbb{I}_{0}$; $\mathbb{I}_{1} \hat{=}\left\{i \mid y_{i}=1,1 \leqslant i \leqslant n\right\}$ and $m_{1}=\sum_{i=1}^{n} I\left(y_{i}=1\right)$ denote the number of elements in $\mathbb{I}_{1}$. The observed-data likelihood function for $\left(\phi_{0}, \phi_{1}, \lambda\right)$ is then given by

$$
\begin{aligned}
& L\left(\phi_{0}, \phi_{1}, \lambda \mid Y_{\text {obs }}\right) \\
= & \left(\phi_{0}+\phi_{2} \mathrm{e}^{-\lambda}\right)^{m_{0}} \times\left(\phi_{1}+\phi_{2} \lambda \mathrm{e}^{-\lambda}\right)^{m_{1}} \\
& \times \phi_{2}^{n-m_{0}-m_{1}} \prod_{i \notin \mathbb{I}_{0} \cup \mathbb{I}_{1}} \frac{\lambda^{y_{i}} \mathrm{e}^{-\lambda}}{y_{i} !},
\end{aligned}
$$

Theorem 4. (Conditional distribution of $\eta \mid Y$ ). Let $\eta \sim$ $\operatorname{Bernoulli}(p)$ and the pmf of $Y$ be specified by (3.12). Based on the SR (2.3), we have

$$
\eta \mid(Y=y) \sim \begin{cases}\operatorname{Bernoulli}\left(p \varphi_{1}\right), & \text { if } y=0 \\ \operatorname{Bernoulli}\left(1-\varphi_{2}+p \varphi_{2}\right), & \text { if } y=1 \\ \operatorname{Bernoulli}(p), & \text { if } y \geqslant 2\end{cases}
$$

where $\varphi_{1}$ and $\varphi_{2}$ are given by (3.14).

Proof. Since $\eta \sim \operatorname{Bernoulli}(p), \eta$ only takes the value 0 or 1 . By the SR (2.3), we have

$$
\operatorname{Pr}(\eta=1 \mid Y=y)
$$

where $\phi_{2}=1-\phi_{0}-\phi_{1}$ and $N=\sum_{i \notin \mathbb{I}_{0} \cup \mathbb{I}_{1}} y_{i}$. To calculate the Fisher information matrix, we need the following results.

Theorem 6. (Expectations). The expectations of $m_{0}, m_{1}$ and $N$ are given by

$$
E\left(m_{0}\right)=n\left(\phi_{0}+\phi_{2} \mathrm{e}^{-\lambda}\right),
$$




$$
\begin{aligned}
E\left(m_{1}\right) & =n\left(\phi_{1}+\phi_{2} \lambda \mathrm{e}^{-\lambda}\right), \\
E(N) & =n \phi_{2} \lambda\left(1-\mathrm{e}^{-\lambda}\right) .
\end{aligned}
$$

Proof. It is easy to show the expressions of $E\left(m_{0}\right)$ and $E\left(m_{1}\right)$, and we have two methods to verify the last one. The first way is to directly calculate $E(N)$ as follows. Since

$$
E\left(Y_{i} \mid Y_{i} \geqslant 2\right)=\frac{\lambda\left(1-\mathrm{e}^{-\lambda}\right)}{1-\mathrm{e}^{-\lambda}-\lambda \mathrm{e}^{-\lambda}}, \quad i \notin \mathbb{I}_{0} \cup \mathbb{I}_{1},
$$

we have

$$
\begin{aligned}
E(N) & =\left[E\left(N \mid m_{0}, m_{1}\right)\right] \\
& =E\left[\frac{\left(n-m_{0}-m_{1}\right) \lambda\left(1-\mathrm{e}^{-\lambda}\right)}{1-\mathrm{e}^{-\lambda}-\lambda \mathrm{e}^{-\lambda}}\right] \\
& =\frac{\left[n-E\left(m_{0}\right)-E\left(m_{1}\right)\right] \lambda\left(1-\mathrm{e}^{-\lambda}\right)}{1-\mathrm{e}^{-\lambda}-\lambda \mathrm{e}^{-\lambda}} \\
& \stackrel{(4.2)}{=} n \phi_{2} \lambda\left(1-\mathrm{e}^{-\lambda}\right) .
\end{aligned}
$$

Alternatively, we noted that

$$
N=\sum_{i \notin \mathbb{I}_{0} \cup \mathbb{I}_{1}} y_{i}=\sum_{i=1}^{n} y_{i}-m_{1} .
$$

Thus, $E(N)=n E\left(Y_{1}\right)-E\left(m_{1}\right) \stackrel{(3.3)}{=} n\left(\phi_{1}+\phi_{2} \lambda\right)-n\left(\phi_{1}+\right.$ $\left.\phi_{2} \lambda \mathrm{e}^{-\lambda}\right)=n \phi_{2} \lambda\left(1-\mathrm{e}^{-\lambda}\right)$.

\subsection{MLEs via the Fisher scoring algorithm}

In this section, we use the Fisher scoring algorithm to calculate the MLEs of $\phi_{0}, \phi_{1}$ and $\lambda$. The score vector $\nabla \ell$ and the Hessian matrix $\nabla^{2} \ell$ are given by

$$
\begin{aligned}
\nabla \ell\left(\phi_{0}, \phi_{1}, \lambda \mid Y_{\text {obs }}\right)= & \left(\frac{\partial \ell}{\partial \phi_{0}}, \frac{\partial \ell}{\partial \phi_{1}}, \frac{\partial \ell}{\partial \lambda}\right)^{\top} \text { and } \\
\nabla^{2} \ell\left(\phi_{0}, \phi_{1}, \lambda \mid Y_{\text {obs }}\right)= & \left(\begin{array}{ccc}
\frac{\partial^{2} \ell}{\partial \phi_{0}^{2}} & \frac{\partial^{2} \ell}{\partial \phi_{0} \partial \phi_{1}} & \frac{\partial^{2} \ell}{\partial \phi_{0} \partial \lambda} \\
\frac{\partial^{2} \ell}{\partial \phi_{1} \partial \phi_{0}} & \frac{\partial^{2} \ell}{\partial \phi_{1}^{2}} & \frac{\partial^{2} \ell}{\partial \phi_{1} \partial \lambda} \\
\frac{\partial^{2} \ell}{\partial \lambda \partial \phi_{0}} & \frac{\partial^{2} \ell}{\partial \lambda \partial \phi_{1}} & \frac{\partial^{2} \ell}{\partial \lambda^{2}}
\end{array}\right),
\end{aligned}
$$

respectively, where

$$
\begin{aligned}
\frac{\partial \ell}{\partial \phi_{0}}= & \frac{m_{0}\left(1-\mathrm{e}^{-\lambda}\right)}{\phi_{0}+\phi_{2} \mathrm{e}^{-\lambda}}-\frac{m_{1} \lambda \mathrm{e}^{-\lambda}}{\phi_{1}+\phi_{2} \lambda \mathrm{e}^{-\lambda}}-\frac{n-m_{0}-m_{1}}{\phi_{2}}, \\
\frac{\partial \ell}{\partial \phi_{1}}= & -\frac{m_{0} \mathrm{e}^{-\lambda}}{\phi_{0}+\phi_{2} \mathrm{e}^{-\lambda}}+\frac{m_{1}\left(1-\lambda \mathrm{e}^{-\lambda}\right)}{\phi_{1}+\phi_{2} \lambda \mathrm{e}^{-\lambda}}-\frac{n-m_{0}-m_{1}}{\phi_{2}}, \\
\frac{\partial \ell}{\partial \lambda}= & -\frac{m_{0} \phi_{2} \mathrm{e}^{-\lambda}}{\phi_{0}+\phi_{2} \mathrm{e}^{-\lambda}}+\frac{m_{1} \phi_{2}(1-\lambda) \mathrm{e}^{-\lambda}}{\phi_{1}+\phi_{2} \lambda \mathrm{e}^{-\lambda}} \\
& -\left(n-m_{0}-m_{1}\right)+\frac{N}{\lambda},
\end{aligned}
$$

and

$$
\begin{aligned}
\frac{\partial^{2} \ell}{\partial \phi_{0}^{2}}= & -\frac{m_{0}\left(1-\mathrm{e}^{-\lambda}\right)^{2}}{\left[\phi_{0}+\phi_{2} \mathrm{e}^{-\lambda}\right]^{2}}-\frac{m_{1} \lambda^{2} \mathrm{e}^{-2 \lambda}}{\left[\phi_{1}+\phi_{2} \lambda \mathrm{e}^{-\lambda}\right]^{2}} \\
& -\frac{n-m_{0}-m_{1}}{\phi_{2}^{2}}, \\
\frac{\partial^{2} \ell}{\partial \phi_{1}^{2}}= & -\frac{m_{0} \mathrm{e}^{-2 \lambda}}{\left[\phi_{0}+\phi_{2} \mathrm{e}^{-\lambda}\right]^{2}}-\frac{m_{1}\left(1-\lambda \mathrm{e}^{-\lambda}\right)^{2}}{\left[\phi_{1}+\phi_{2} \lambda \mathrm{e}^{-\lambda}\right]^{2}} \\
& -\frac{n-m_{0}-m_{1}}{\phi_{2}^{2}}, \\
\frac{\partial^{2} \ell}{\partial \lambda^{2}}= & \frac{m_{0} \phi_{0} \phi_{2} \mathrm{e}^{-\lambda}}{\left[\phi_{0}+\phi_{2} \mathrm{e}^{-\lambda}\right]^{2}} \\
& +\frac{m_{1} \phi_{2} \mathrm{e}^{-\lambda}\left[\phi_{1}(\lambda-2)-\phi_{2} \mathrm{e}^{-\lambda}\right]}{\left[\phi_{1}+\phi_{2} \lambda \mathrm{e}^{-\lambda}\right]^{2}}-\frac{N}{\lambda^{2}} \\
\frac{\partial^{2} \ell}{\partial \phi_{0} \partial \phi_{1}}= & \frac{m_{0} \mathrm{e}^{-\lambda}\left(1-\mathrm{e}^{-\lambda}\right)}{\left[\phi_{0}+\phi_{2} \mathrm{e}^{-\lambda}\right]^{2}}+\frac{m_{1} \lambda \mathrm{e}^{-\lambda}\left(1-\lambda \mathrm{e}^{-\lambda}\right)}{\left[\phi_{1}+\phi_{2} \lambda \mathrm{e}^{-\lambda}\right]^{2}} \\
& -\frac{n-m_{0}-m_{1}}{\phi_{2}^{2}}, \\
\frac{\partial^{2} \ell}{\partial \phi_{0} \partial \lambda}= & \frac{m_{0}\left(1-\phi_{1}\right) \mathrm{e}^{-\lambda}}{\left[\phi_{0}+\phi_{2} \mathrm{e}^{-\lambda}\right]^{2}}-\frac{m_{1} \phi_{1}(1-\lambda) \mathrm{e}^{-\lambda}}{\left[\phi_{1}+\phi_{2} \lambda \mathrm{e}^{-\lambda}\right]^{2}} \\
\frac{\partial^{2} \ell}{\partial \phi_{1} \partial \lambda}= & \frac{m_{0} \phi_{0} \mathrm{e}^{-\lambda}}{\left[\phi_{0}+\phi_{2} \mathrm{e}^{-\lambda}\right]^{2}}-\frac{m_{1}\left(1-\phi_{0}\right)(1-\lambda) \mathrm{e}^{-\lambda}}{\left[\phi_{1}+\phi_{2} \lambda \mathrm{e}^{-\lambda}\right]^{2}}
\end{aligned}
$$

Thus, by utilizing (4.2), we can calculate the Fisher information as follows:

$$
J\left(\phi_{0}, \phi_{1}, \lambda\right)=E\left[-\nabla^{2} \ell\left(\phi_{0}, \phi_{1}, \lambda \mid Y_{\text {obs }}\right)\right] .
$$

Let $\left(\phi_{0}^{(0)}, \phi_{1}^{(0)}, \lambda^{(0)}\right)$ be initial values of the MLEs $\left(\hat{\phi}_{0}, \hat{\phi}_{1}, \hat{\lambda}\right)$. If $\left(\phi_{0}^{(t)}, \phi_{1}^{(t)}, \lambda^{(t)}\right)$ denote the $t$-th approximations of $\left(\hat{\phi}_{0}, \hat{\phi}_{1}, \hat{\lambda}\right)$, then their $(t+1)$-th approximation can be obtained by the following Fisher scoring algorithm:

$$
\begin{aligned}
\left(\begin{array}{c}
\phi_{0}^{(t+1)} \\
\phi_{1}^{(t+1)} \\
\lambda^{(t+1)}
\end{array}\right)= & \left(\begin{array}{c}
\phi_{0}^{(t)} \\
\phi_{1}^{(t)} \\
\lambda^{(t)}
\end{array}\right) \\
& +J^{-1}\left(\phi_{0}^{(t)}, \phi_{1}^{(t)}, \lambda^{(t)}\right) \nabla \ell\left(\phi_{0}^{(t)}, \phi_{1}^{(t)}, \lambda^{(t)} \mid Y_{\mathrm{obs}}\right)
\end{aligned}
$$

As a by-product, the standard errors of the MLEs $\left(\hat{\phi}_{0}, \hat{\phi}_{1}, \hat{\lambda}\right)$ are the square roots of the diagonal elements $J^{k k}$ of the inverse Fisher information matrix $J^{-1}\left(\hat{\phi}_{0}, \hat{\phi}_{1}, \hat{\lambda}\right)$. Thus, the $(1-\alpha) 100 \%$ asymptotic Wald confidence intervals (CIs) of $\phi_{0}, \phi_{1}$ and $\lambda$ are given by

$$
\begin{array}{ll}
{\left[\hat{\phi}_{k-1}-z_{\alpha / 2} \sqrt{J^{k k}},\right.} & \left.\hat{\phi}_{k-1}+z_{\alpha / 2} \sqrt{J^{k k}}\right], \quad k=1,2, \\
{\left[\hat{\lambda}-z_{\alpha / 2} \sqrt{J^{33}},\right.} & \left.\hat{\lambda}+z_{\alpha / 2} \sqrt{J^{33}}\right],
\end{array}
$$

respectively, where $z_{\alpha}$ denotes the $\alpha$-th upper quantile of the standard normal distribution. 


\subsection{MLEs via the EM algorithm}

The zero observations from a ZOIP distribution can be categorized into the extra zeros resulted from the degenerate distribution at zero because of population variability at the point zero and the structural zeros came from an ordinary Poisson distribution. The one observations can similarly be classified into the extra ones resulted from the degenerate distribution at one because of population variability at the point one and structural ones came from an ordinary Poisson distribution. Thus, we partition

$$
\mathbb{I}_{0}=\mathbb{I}_{0}^{\text {extra }} \cup \mathbb{I}_{0}^{\text {structral }} \quad \text { and } \quad \mathbb{I}_{1}=\mathbb{I}_{1}^{\text {extra }} \cup \mathbb{I}_{1}^{\text {structral }} .
$$

Note that a major obstacle from obtaining explicit solutions of MLEs of parameters from (4.1) is the first and second terms in the right-hand-side of (4.1). To overcome this difficulty, we augment $Y_{\text {obs }}$ with two latent variables $W_{0}$ and $W_{1}$, where $W_{0}$ denotes the number of $\mathbb{I}_{0}^{\text {extra }}$ to split $m_{0}$ into $W_{0}$ and $m_{0}-W_{0}$ and $W_{1}$ denotes the number of $\mathbb{I}_{1}^{\text {extra }}$ to split $m_{1}$ into $W_{1}$ and $m_{1}-W_{1}$. Thus, the resulting conditional predictive distributions of $W_{0}$ and $W_{1}$ given $\left(Y_{\mathrm{obs}}, \phi_{0}, \phi_{1}, \lambda\right)$ are given by

$W_{0} \mid\left(Y_{\mathrm{obs}}, \phi_{0}, \phi_{1}, \lambda\right) \sim \operatorname{Binomial}\left(m_{0}, \frac{\phi_{0}}{\phi_{0}+\phi_{2} \mathrm{e}^{-\lambda}}\right) \quad$ and

$W_{1} \mid\left(Y_{\mathrm{obs}}, \phi_{0}, \phi_{1}, \lambda\right) \sim \operatorname{Binomial}\left(m_{1}, \frac{\phi_{1}}{\phi_{1}+\phi_{2} \lambda \mathrm{e}^{-\lambda}}\right)$,

respectively. On the other hand, the complete-data likelihood function is proportional to

$$
\begin{aligned}
& L\left(\phi_{0}, \phi_{1}, \lambda \mid Y_{\text {com }}\right) \\
\propto & \phi_{0}^{w_{0}}\left[\left(1-\phi_{0}-\phi_{1}\right) \mathrm{e}^{-\lambda}\right]^{m_{0}-w_{0}} \\
& \times \phi_{1}^{w_{1}}\left[\left(1-\phi_{0}-\phi_{1}\right) \lambda \mathrm{e}^{-\lambda}\right]^{m_{1}-w_{1}} \\
& \times\left(1-\phi_{0}-\phi_{1}\right)^{n-m_{0}-m_{1}} \mathrm{e}^{-\left(n-m_{0}-m_{1}\right) \lambda} \lambda^{N} \\
= & \phi_{0}^{w_{0}} \phi_{1}^{w_{1}}\left(1-\phi_{0}-\phi_{1}\right)^{n-w_{0}-w_{1}} \mathrm{e}^{-\left(n-w_{0}-w_{1}\right) \lambda} \lambda^{m_{1}-w_{1}+N} .
\end{aligned}
$$

Thus the M-step is to find the complete-data MLEs

$$
\begin{aligned}
\hat{\phi}_{0} & =\frac{w_{0}}{n}, \quad \hat{\phi}_{1}=\frac{w_{1}}{n} \text { and } \\
\hat{\lambda} & =\frac{N+m_{1}-w_{1}}{n-w_{0}-w_{1}}=\frac{N+m_{1}-n \hat{\phi}_{1}}{n\left(1-\hat{\phi}_{0}-\hat{\phi}_{1}\right)} .
\end{aligned}
$$

The E-step is to replace $w_{0}$ and $w_{1}$ in (4.9) by their conditional expectations:

$$
\begin{aligned}
& E\left(W_{0} \mid Y_{\mathrm{obs}}, \phi_{0}, \phi_{1}, \lambda\right)=\frac{m_{0} \phi_{0}}{\phi_{0}+\left(1-\phi_{0}-\phi_{1}\right) \mathrm{e}^{-\lambda}} \text { and } \\
& E\left(W_{1} \mid Y_{\mathrm{obs}}, \phi_{0}, \phi_{1}, \lambda\right)=\frac{m_{1} \phi_{1}}{\phi_{1}+\left(1-\phi_{0}-\phi_{1}\right) \lambda \mathrm{e}^{-\lambda}}
\end{aligned}
$$

\subsection{Bootstrap confidence intervals}

The Wald CIs of $\phi_{0}$ and $\phi_{1}$ specified by (4.6) may fall outside the unit interval $[0,1)$. The Wald CI of $\lambda$ given by (4.6) is reliable only for large sample sizes and its lower bound may be less than 0. For small sample sizes, the bootstrap method is a useful tool to find a bootstrap CI for an arbitrary function of $\phi_{0}, \phi_{1}$ and $\lambda$, say, $\vartheta=h\left(\phi_{0}, \phi_{1}, \lambda\right)$. Let $\hat{\vartheta}=h\left(\hat{\phi}_{0}, \hat{\phi}_{1}, \hat{\lambda}\right)$ denote the MLE of $\vartheta$, where $\left(\hat{\phi}_{0}, \hat{\phi}_{1}, \hat{\lambda}\right)$ represent the MLEs of $\left(\phi_{0}, \phi_{1}, \lambda\right)$ calculated by either the Fisher scoring algorithm (4.5) or the EM algorithm (4.9)-(4.10). Based on the obtained MLEs $\left(\hat{\phi}_{0}, \hat{\phi}_{1}, \hat{\lambda}\right)$, by using the SR (2.1) we can generate $Y_{1}^{*}, \ldots, Y_{n}^{*} \stackrel{\text { iid }}{\sim} \operatorname{ZOIP}\left(\hat{\phi}_{0}, \hat{\phi}_{1}, \hat{\lambda}\right)$. Having obtained $Y_{\text {obs }}^{*}=\left\{y_{1}^{*}, \ldots, y_{n}^{*}\right\}$, we can calculate the bootstrap replications $\left(\hat{\phi}_{0}^{*}, \hat{\phi}_{1}^{*}, \hat{\lambda}^{*}\right)$ and get $\hat{\vartheta}^{*}=h\left(\hat{\phi}_{0}^{*}, \hat{\phi}_{1}^{*}, \hat{\lambda}^{*}\right)$. Independently repeating this process $G$ times, we obtain $G$ bootstrap replications $\left\{\hat{\vartheta}_{g}^{*}\right\}_{g=1}^{G}$. Consequently, the standard error, se $(\hat{\vartheta})$, of $\hat{\vartheta}$ can be estimated by the sample standard deviation of the $G$ replications, i.e.,

$$
\widehat{\operatorname{se}}(\hat{\vartheta})=\left\{\frac{1}{G-1} \sum_{g=1}^{G}\left[\hat{\vartheta}_{g}^{*}-\left(\hat{\vartheta}_{1}^{*}+\cdots+\hat{\vartheta}_{G}^{*}\right) / G\right]^{2}\right\}^{1 / 2} .
$$

If $\left\{\hat{\vartheta}_{g}^{*}\right\}_{g=1}^{G}$ is approximately normally distributed, the first $(1-\alpha) 100 \%$ bootstrap CI for $\vartheta$ is

$$
\left[\hat{\vartheta}-z_{\alpha / 2} \cdot \widehat{\operatorname{se}}(\hat{\vartheta}), \hat{\vartheta}+z_{\alpha / 2} \cdot \widehat{\operatorname{se}}(\hat{\vartheta})\right] .
$$

Alternatively, if $\left\{\hat{\vartheta}_{g}^{*}\right\}_{g=1}^{G}$ is non-normally distributed, the second $(1-\alpha) 100 \%$ bootstrap CI of $\vartheta$ is given by

$$
\left[\hat{\vartheta}_{\mathrm{L}}, \hat{\vartheta}_{\mathrm{U}}\right]
$$

where $\hat{\vartheta}_{\mathrm{L}}$ and $\hat{\vartheta}_{\mathrm{U}}$ are the $100(\alpha / 2)$ and $100(1-\alpha / 2)$ percentiles of $\left\{\hat{\vartheta}_{g}^{*}\right\}_{g=1}^{G}$, respectively.

\section{TESTING HYPOTHESES WITH LARGE SAMPLE SIZES}

In this section, we consider the testing hypotheses for (i) $H_{0}:\left(\phi_{0}, \phi_{1}\right)=(0,0)$; (ii) $H_{0}: \phi_{1}=0$; (iii) $H_{0}: \phi_{0}=0$; (iv) $H_{0}: \lambda=\lambda_{0}$. For (i), when $H_{0}$ is true, since the parameter values are located at the vertex boundary of the bounded parameter space, the traditional asymptotic property of LRT is not applicable. Therefore, we only consider the score test. For (ii)-(iii), although the parameter value is still on the boundary of the parameter space, we can provide appropriate reference distribution. Finally for (iv), it is a standard two-sided test.

\subsection{Score test for simultaneous zero-and-one inflation}

First, we develop a score test to examine whether there exist excessive zeros and excessive ones in the observations simultaneously, i.e., zero-and-one inflation in the ZOIP 
model. The null and alternative hypotheses are as follows (5.1)

$$
H_{0}:\left(\phi_{0}, \phi_{1}\right)=(0,0) \text { against } H_{1}:\left(\phi_{0}, \phi_{1}\right) \neq(0,0) \text {. }
$$

By reparametrization, three new parameters are introduced as follows:

$$
\theta_{0}=\frac{\phi_{0}}{1-\phi_{0}-\phi_{1}}, \quad \theta_{1}=\frac{\phi_{1}}{1-\phi_{0}-\phi_{1}} \quad \text { and } \quad \beta=\log \lambda .
$$

Then, testing $H_{0}$ specified in (5.1) is equivalent to testing $H_{0}^{*}:\left(\theta_{0}, \theta_{1}\right)=(0,0)$. The observed-data log-likelihood function now becomes

$$
\begin{aligned}
\ell_{1} \hat{=} & \ell_{1}\left(\theta_{0}, \theta_{1}, \beta\right)=\sum_{i=1}^{n}\left\{-\log \left(1+\theta_{0}+\theta_{1}\right)\right. \\
& +\left[\log \left(\theta_{0}+\mathrm{e}^{-\lambda}\right)\right] I\left(y_{i}=0\right) \\
& +\left[\log \left(\theta_{1}+\lambda \mathrm{e}^{-\lambda}\right)\right] I\left(y_{i}=1\right) \\
& \left.+\left[y_{i} \log \lambda-\lambda-\log \left(y_{i} !\right)\right] I\left(y_{i} \geqslant 2\right)\right\} .
\end{aligned}
$$

The score vector is now denoted by

$$
U\left(\theta_{0}, \theta_{1}, \beta\right)=\left(\frac{\partial \ell_{1}}{\partial \theta_{0}}, \frac{\partial \ell_{1}}{\partial \theta_{1}}, \frac{\partial \ell_{1}}{\partial \beta}\right)^{\top},
$$

where

$$
\begin{aligned}
\frac{\partial \ell_{1}}{\partial \theta_{0}}= & \sum_{i=1}^{n}\left[-\frac{1}{1+\theta_{0}+\theta_{1}}+\frac{1}{\theta_{0}+\mathrm{e}^{-\lambda}} I\left(y_{i}=0\right)\right], \\
\frac{\partial \ell_{1}}{\partial \theta_{1}}= & \sum_{i=1}^{n}\left[-\frac{1}{1+\theta_{0}+\theta_{1}}+\frac{1}{\theta_{1}+\lambda \mathrm{e}^{-\lambda}} I\left(y_{i}=1\right)\right], \\
\frac{\partial \ell_{1}}{\partial \beta}= & \sum_{i=1}^{n}\left[-\frac{\lambda \mathrm{e}^{-\lambda}}{\theta_{0}+\mathrm{e}^{-\lambda}} I\left(y_{i}=0\right)\right. \\
& \left.-\frac{\left(\lambda^{2}-\lambda\right) \mathrm{e}^{-\lambda}}{\theta_{1}+\lambda \mathrm{e}^{-\lambda}} I\left(y_{i}=1\right)+\left(y_{i}-\lambda\right) I\left(y_{i} \geqslant 2\right)\right] .
\end{aligned}
$$

The second derivatives are given by

$$
\begin{aligned}
\frac{\partial^{2} \ell_{1}}{\partial \theta_{0}^{2}}= & \sum_{i=1}^{n}\left[\frac{1}{\left(1+\theta_{0}+\theta_{1}\right)^{2}}-\frac{1}{\left(\theta_{0}+\mathrm{e}^{-\lambda}\right)^{2}} I\left(y_{i}=0\right)\right], \\
\frac{\partial^{2} \ell_{1}}{\partial \theta_{1}^{2}}= & \sum_{i=1}^{n}\left[\frac{1}{\left(1+\theta_{0}+\theta_{1}\right)^{2}}\right. \\
& \left.-\frac{1}{\left(\theta_{1}+\lambda \mathrm{e}^{-\lambda}\right)^{2}} I\left(y_{i}=1\right)\right], \\
\frac{\partial^{2} \ell_{1}}{\partial \beta^{2}}= & \sum_{i=1}^{n}\left\{-\frac{\lambda \mathrm{e}^{-\lambda}\left[\theta_{0}(1-\lambda)+\mathrm{e}^{-\lambda}\right]}{\left(\theta_{0}+\mathrm{e}^{-\lambda}\right)^{2}} I\left(y_{i}=0\right)\right. \\
& -\frac{\left[\theta_{1}\left(3 \lambda^{2}-\lambda-\lambda^{3}\right) \mathrm{e}^{-\lambda}+\lambda^{3} \mathrm{e}^{-2 \lambda}\right]}{\left(\theta_{1}+\lambda \mathrm{e}^{-\lambda}\right)^{2}} I\left(y_{i}=1\right) \\
& \left.-\lambda I\left(y_{i} \geqslant 2\right)\right\},
\end{aligned}
$$

$\frac{\partial^{2} \ell_{1}}{\partial \theta_{0} \partial \theta_{1}}=\sum_{i=1}^{n} \frac{1}{\left(1+\theta_{0}+\theta_{1}\right)^{2}}$

$\frac{\partial^{2} \ell_{1}}{\partial \theta_{0} \partial \beta}=\sum_{i=1}^{n}\left[\frac{\lambda \mathrm{e}^{-\lambda}}{\left(\theta_{0}+\mathrm{e}^{-\lambda}\right)^{2}} I\left(y_{i}=0\right)\right]$,

$\frac{\partial^{2} \ell_{1}}{\partial \theta_{1} \partial \beta}=\sum_{i=1}^{n}\left[\frac{\left(\lambda^{2}-\lambda\right) \mathrm{e}^{-\lambda}}{\left(\theta_{1}+\lambda \mathrm{e}^{-\lambda}\right)^{2}} I\left(y_{i}=1\right)\right]$.

Since

$$
\begin{aligned}
E\left[I\left(y_{i}=0\right)\right] & =\frac{\theta_{0}+\mathrm{e}^{-\lambda}}{1+\theta_{0}+\theta_{1}}, \\
E\left[I\left(y_{i}=1\right)\right] & =\frac{\theta_{1}+\lambda \mathrm{e}^{-\lambda}}{1+\theta_{0}+\theta_{1}} \text { and } \\
E\left[I\left(y_{i} \geqslant 2\right)\right] & =\frac{1-\mathrm{e}^{-\lambda}-\lambda \mathrm{e}^{-\lambda}}{1+\theta_{0}+\theta_{1}},
\end{aligned}
$$

the Fisher information matrix can be calculated as $J\left(\theta_{0}, \theta_{1}, \beta\right)=\left(J_{j j^{\prime}}\right)$, where

$$
\begin{aligned}
J_{11}= & -E\left(\frac{\partial^{2} \ell_{1}}{\partial \theta_{0}^{2}}\right)=\frac{n\left(1+\theta_{1}-\mathrm{e}^{-\lambda}\right)}{\left(1+\theta_{0}+\theta_{1}\right)^{2}\left(\theta_{0}+\mathrm{e}^{-\lambda}\right)}, \\
J_{22}= & -E\left(\frac{\partial^{2} \ell_{1}}{\partial \theta_{1}^{2}}\right)=\frac{n\left(1+\theta_{0}-\lambda \mathrm{e}^{-\lambda}\right)}{\left(1+\theta_{0}+\theta_{1}\right)^{2}\left(\theta_{1}+\lambda \mathrm{e}^{-\lambda}\right)}, \\
J_{33}= & -E\left(\frac{\partial^{2} \ell_{1}}{\partial \beta^{2}}\right)=\frac{n \lambda \mathrm{e}^{-\lambda}\left[\theta_{0}(1-\lambda)+\mathrm{e}^{-\lambda}\right]}{\left(\theta_{0}+\mathrm{e}^{-\lambda}\right)\left(1+\theta_{0}+\theta_{1}\right)} \\
& +\frac{n\left[\theta_{1}\left(3 \lambda^{2}-\lambda-\lambda^{3}\right) \mathrm{e}^{-\lambda}+\lambda^{3} \mathrm{e}^{-2 \lambda}\right]}{\left(\theta_{1}+\lambda \mathrm{e}^{-\lambda}\right)\left(1+\theta_{0}+\theta_{1}\right)} \\
& +\frac{n \lambda\left(1-\mathrm{e}^{\lambda}-\lambda \mathrm{e}^{\lambda}\right)}{1+\theta_{0}+\theta_{1}}, \\
J_{12}= & -E\left(\frac{\partial^{2} \ell_{1}}{\partial \theta_{0} \partial \theta_{1}}\right)=-\frac{n}{\left(1+\theta_{0}+\theta_{1}\right)^{2}}, \\
J_{13}= & -E\left(\frac{\partial^{2} \ell_{1}}{\partial \theta_{0} \partial \beta}\right)=-\frac{n \lambda \mathrm{e}^{-\lambda}}{\left(1+\theta_{0}+\theta_{1}\right)\left(\theta_{0}+\mathrm{e}^{-\lambda}\right)}, \\
J_{23}= & -E\left(\frac{\partial^{2} \ell_{1}}{\partial \theta_{1} \partial \beta}\right)=-\frac{n\left(\lambda^{2}-\lambda\right) \mathrm{e}^{-\lambda}}{\left(1+\theta_{0}+\theta_{1}\right)\left(\theta_{1}+\lambda \mathrm{e}^{-\lambda}\right)} .
\end{aligned}
$$

Under $H_{0}^{*}$, the score test statistic

$$
T_{1}=U^{\top}(0,0, \hat{\beta}) J^{-1}(0,0, \hat{\beta}) U(0,0, \hat{\beta}) \dot{\sim} \chi^{2}(2),
$$

where $\hat{\beta}=\log (\bar{y})$ and $U(0,0, \hat{\beta})=\left(m_{0} \mathrm{e}^{\bar{y}}-n, m_{1} \mathrm{e}^{\bar{y}} / \bar{y}-\right.$ $n, 0)^{\top}$. The $p$-value is

$$
p_{\mathrm{v} 1}=\operatorname{Pr}\left(T_{1}>t_{1} \mid H_{0}^{*}\right)=\operatorname{Pr}\left(\chi^{2}(2)>t_{1}\right),
$$

where $t_{1}$ is the observed value of $T_{1}$.

\subsection{Likelihood ratio test for one inflation}

If the null hypothesis $H_{0}$ specified by (5.1) is rejected at the $\alpha$ level of significance (i.e, at least one of the $\phi_{0}$ and $\phi_{1}$ are positive), we could next test whether there exist extra ones in the observations, i.e., one-inflation in the ZOIP 
model. We consider the following null and alternative hypotheses

$$
H_{0}: \phi_{1}=0 \text { against } H_{1}: \phi_{1}>0 \text {. }
$$

Under $H_{0}$, the likelihood ratio (LR) test statistic is given by

$$
T_{2}=-2\left\{\ell\left(\hat{\phi}_{0, H_{0}}, 0, \hat{\lambda}_{H_{0}} \mid Y_{\mathrm{obs}}\right)-\ell\left(\hat{\phi}_{0}, \hat{\phi}_{1}, \hat{\lambda} \mid Y_{\mathrm{obs}}\right)\right\}
$$

where $\left(\hat{\phi}_{0, H_{0}}, \hat{\lambda}_{H_{0}}\right)$ denote the constrained MLEs of $\left(\phi_{0}, \lambda\right)$ under $H_{0}$ and $\left(\hat{\phi}_{0}, \hat{\phi}_{1}, \hat{\lambda}\right)$ denote the unconstrained MLEs of $\left(\phi_{0}, \phi_{1}, \lambda\right)$, which are obtained by either the Fisher scoring algorithm (4.5) or the EM algorithm (4.9)-(4.10). Note that under $H_{0}$, the $\operatorname{ZOIP}\left(\phi_{0}, \phi_{1} ; \lambda\right)$ distribution reduces to the zero-inflated Poisson distribution $\operatorname{ZIP}\left(\phi_{0}, \lambda\right)$. Thus, the MLEs of $\left(\phi_{0}, \lambda\right)$ can be calculated by the following EM iteration:

$$
\begin{aligned}
\phi_{0, H_{0}}^{(t+1)} & =\frac{m_{0} \phi_{0, H_{0}}^{(t)}}{n\left[\phi_{0, H_{0}}^{(t)}+\left(1-\phi_{0, H_{0}}^{(t)}\right) \mathrm{e}^{\left.-\lambda_{H_{0}}^{(t+1)}\right]}\right]}, \\
\lambda_{H_{0}}^{(t+1)} & =\frac{\bar{y}}{1-\phi_{0, H_{0}}^{(t)}},
\end{aligned}
$$

where $\bar{y}=(1 / n) \sum_{i=1}^{n} y_{i}$.

Standard large-sample theory suggests that the asymptotic null distribution of $T_{2}$ is $\chi^{2}(1)$. However, the null hypothesis in (5.5) corresponds to $\phi_{1}$ being on the boundary of the parameter space and the appropriate null distribution is a 50:50 mixture of $\chi^{2}(0)$ (i.e., Degenerate $\left.(0)\right)$ and $\chi^{2}(1)$, see Self and Liang [28] and Feng and McCulloch [9]. Hence, the corresponding $p$-value (Jansakul and Hinde, [14], p. 78; Joe and Zhu, [15], p. 225) is

$$
p_{\mathrm{v} 2}=\operatorname{Pr}\left(T_{2}>t_{2} \mid H_{0}\right)=\frac{1}{2} \operatorname{Pr}\left(\chi^{2}(1)>t_{2}\right) .
$$

\subsection{Score test for one inflation}

Alternatively, the score test can be used for testing $H_{0}$ specified in (5.5), which is equivalent to testing $H_{0}^{*}: \theta_{1}=0$. Let $\left(\theta_{0}, \theta_{1}, \beta\right)$ be defined by $(5.2)$. Under $H_{0}^{*}$, the score test statistic

$$
T_{3}=U^{\top}\left(\hat{\theta}_{0}, 0, \hat{\beta}\right) J^{-1}\left(\hat{\theta}_{0}, 0, \hat{\beta}\right) U\left(\hat{\theta}_{0}, 0, \hat{\beta}\right) \dot{\sim} \chi^{2}(1),
$$

where $\hat{\theta}_{0}=\hat{\phi}_{0, H_{0}} /\left(1-\hat{\phi}_{0, H_{0}}\right)$ and $\hat{\beta}=\log \left(\hat{\lambda}_{H_{0}}\right)$ denote the MLEs of $\theta_{0}$ and $\beta$ under $H_{0}^{*}$, and $\left(\hat{\phi}_{0, H_{0}}, \hat{\lambda}_{H_{0}}\right)$ are determined by (5.7). Note that the score vector $U\left(\theta_{0}, \theta_{1}, \beta\right)$ evaluated at $\left(\theta_{0}, \theta_{1}, \beta\right)=\left(\hat{\theta}_{0}, 0, \hat{\beta}\right)$ is given by

$$
U\left(\hat{\theta}_{0}, 0, \hat{\beta}\right)=\left(0,-\frac{n}{1+\hat{\theta}_{0}}+\frac{m_{1}}{\hat{\lambda}_{H_{0}} \mathrm{e}^{-\hat{\lambda}_{H_{0}}}}, 0\right)^{\top},
$$

where $m_{1}=\sum_{i=1}^{n} I\left(y_{i}=1\right)$. The corresponding $p$-value is

$$
p_{\mathrm{v} 3}=\operatorname{Pr}\left(T_{3}>t_{3} \mid H_{0}^{*}\right)=\operatorname{Pr}\left(\chi^{2}(1)>t_{3}\right) \text {. }
$$

\subsection{LR test for zero inflation}

To test whether there exist extra zeros in the observations, i.e., zero-inflation in the ZOIP model, we consider the following null and alternative hypotheses

$$
H_{0}: \phi_{0}=0 \text { against } H_{1}: \phi_{0}>0 \text {. }
$$

Under $H_{0}$, the LR test statistic (Jansakul and Hinde, [14], p. 78; Joe and Zhu, [15], p. 225)

$$
\begin{aligned}
T_{4} & =-2\left\{\ell\left(0, \hat{\phi}_{1, H_{0}}, \hat{\lambda}_{H_{0}} \mid Y_{\mathrm{obs}}\right)-\ell\left(\hat{\phi}_{0}, \hat{\phi}_{1}, \hat{\lambda} \mid Y_{\mathrm{obs}}\right)\right\} \\
& \dot{\sim} 0.5 \chi^{2}(0)+0.5 \chi^{2}(1),
\end{aligned}
$$

where $\left(\hat{\phi}_{1, H_{0}}, \hat{\lambda}_{H_{0}}\right)$ denote the constrained MLEs of $\left(\phi_{1}, \lambda\right)$ under $H_{0}$ and $\left(\hat{\phi}_{0}, \hat{\phi}_{1}, \hat{\lambda}\right)$ denote the unconstrained MLEs of $\left(\phi_{0}, \phi_{1}, \lambda\right)$, which are obtained by either the Fisher scoring algorithm (4.5) or the EM algorithm (4.9)-(4.10). Note that under $H_{0}$, the $\operatorname{ZOIP}\left(\phi_{0}, \phi_{1} ; \lambda\right)$ distribution reduces to the one-inflated Poisson distribution $\operatorname{OIP}\left(\phi_{1}, \lambda\right)$. Thus, the MLEs of $\left(\phi_{1}, \lambda\right)$ can be calculated by the following EM iteration:

$$
\begin{aligned}
\phi_{1, H_{0}}^{(t+1)} & =\frac{m_{1} \phi_{1, H_{0}}^{(t)}}{n\left[\phi_{1, H_{0}}^{(t)}+\left(1-\phi_{1, H_{0}}^{(t)}\right) \lambda_{H_{0}}^{(t+1)} \mathrm{e}^{\left.-\lambda_{H_{0}}^{(t+1)}\right]}\right.} \\
\lambda_{H_{0}}^{(t+1)} & =\frac{\bar{y}-\phi_{1, H_{0}}^{(t)}}{1-\phi_{1, H_{0}}^{(t)}}
\end{aligned}
$$

where $\bar{y}=(1 / n) \sum_{i=1}^{n} y_{i}$. Hence the corresponding $p$-value is

$$
p_{\mathrm{v} 4}=\operatorname{Pr}\left(T_{4}>t_{4} \mid H_{0}\right)=\frac{1}{2} \operatorname{Pr}\left(\chi^{2}(1)>t_{4}\right)
$$

\subsection{Score test for zero inflation}

Let $\left(\theta_{0}, \theta_{1}, \beta\right)$ be defined in $(5.2)$, testing $H_{0}$ specified by (5.11) is equivalent to testing $H_{0}^{*}: \theta_{0}=0$. Under $H_{0}^{*}$, the score test statistic

$$
T_{5}=U^{\top}\left(0, \hat{\theta}_{1}, \hat{\beta}\right) J^{-1}\left(0, \hat{\theta}_{1}, \hat{\beta}\right) U\left(0, \hat{\theta}_{1}, \hat{\beta}\right) \dot{\sim} \chi^{2}(1)
$$

where $\hat{\theta}_{1}=\hat{\phi}_{1, H_{0}} /\left(1-\hat{\phi}_{1, H_{0}}\right)$ and $\hat{\beta}=\log \left(\hat{\lambda}_{H_{0}}\right)$ denote the MLEs of $\theta_{1}$ and $\beta$ under $H_{0}^{*}$, and $\left(\hat{\phi}_{1, H_{0}}, \hat{\lambda}_{H_{0}}\right)$ are determined by (5.13). Note that the score vector $U\left(\theta_{0}, \theta_{1}, \beta\right)$ evaluated at $\left(\theta_{0}, \theta_{1}, \beta\right)=\left(0, \hat{\theta}_{1}, \hat{\beta}\right)$ is given by

$$
U\left(0, \hat{\theta}_{1}, \hat{\beta}\right)=\left(-\frac{n}{1+\hat{\theta}_{1}}+m_{0} \mathrm{e}^{\hat{\lambda}_{H_{0}}}, 0,0\right)^{\top},
$$

where $m_{0}=\sum_{i=1}^{n} I\left(y_{i}=0\right)$. The corresponding $p$-value is

$$
p_{\mathrm{v} 5}=\operatorname{Pr}\left(T_{5}>t_{5} \mid H_{0}^{*}\right)=\operatorname{Pr}\left(\chi^{2}(1)>t_{5}\right) .
$$




\subsection{LR test for testing $\lambda=\lambda_{0}$}

Suppose that we want to test the null hypothesis

$$
H_{0}: \lambda=\lambda_{0} \quad \text { against } \quad H_{1}: \lambda \neq \lambda_{0} .
$$

Under $H_{0}$, the LR test statistic

$$
\begin{aligned}
T_{6}= & -2\left\{\ell\left(\hat{\phi}_{0, H_{0}}, \hat{\phi}_{1, H_{0}}, \lambda_{0} \mid Y_{\mathrm{obs}}\right)\right. \\
& \left.-\ell\left(\hat{\phi}_{0}, \hat{\phi}_{1}, \hat{\lambda} \mid Y_{\mathrm{obs}}\right)\right\} \dot{\sim} \chi^{2}(1),
\end{aligned}
$$

where $\left(\hat{\phi}_{0}, \hat{\phi}_{1}, \hat{\lambda}\right)$ are the unconstrained MLEs of $\left(\phi_{0}, \phi_{1}, \lambda\right)$ and $\left(\hat{\phi}_{0, H_{0}}, \hat{\phi}_{1, H_{0}}\right)$ are the restricted MLEs of $\left(\phi_{0}, \phi_{1}\right)$ under $H_{0}$, which can be obtained by the following EM iteration:

$$
\begin{aligned}
\phi_{0, H_{0}}^{(t+1)} & =\frac{m_{0} \phi_{0, H_{0}}^{(t)}}{n\left[\phi_{0, H_{0}}^{(t)}+\left(1-\phi_{0, H_{0}}^{(t)}-\phi_{1, H_{0}}^{(t)}\right) \mathrm{e}^{-\lambda_{0}}\right]}, \\
\phi_{1, H_{0}}^{(t+1)} & =\frac{m_{1} \phi_{1, H_{0}}^{(t)}}{n\left[\phi_{1, H_{0}}^{(t)}+\left(1-\phi_{0, H_{0}}^{(t)}-\phi_{1, H_{0}}^{(t)}\right) \lambda_{0} \mathrm{e}^{-\lambda_{0}}\right]} .
\end{aligned}
$$

The corresponding $p$-value is

$$
p_{\mathrm{v} 6}=\operatorname{Pr}\left(T_{6}>t_{6} \mid H_{0}\right)=\operatorname{Pr}\left(\chi^{2}(1)>t_{6}\right) .
$$

\subsection{Score test for testing $\lambda=\lambda_{0}$}

Let $\left(\theta_{0}, \theta_{1}, \beta\right)$ be defined in $(5.2)$, then, testing $H_{0}$ specified by (5.17) is equivalent to testing $H_{0}^{*}: \beta=\beta_{0}=\log \lambda_{0}$. Under $H_{0}^{*}$, the score test statistic

$$
T_{7}=U^{\top}\left(\hat{\theta}_{0}, \hat{\theta}_{1}, \beta_{0}\right) J^{-1}\left(\hat{\theta}_{0}, \hat{\theta}_{1}, \beta_{0}\right) U\left(\hat{\theta}_{0}, \hat{\theta}_{1}, \beta_{0}\right) \dot{\sim} \chi^{2}(1),
$$

where $\left(\hat{\theta}_{0}, \hat{\theta}_{1}\right)$ are the MLEs of $\left(\theta_{0}, \theta_{1}\right)$ under $H_{0}^{*}$. Hence the $p$-value is

$$
p_{\mathrm{v} 7}=\operatorname{Pr}\left(T_{7}>t_{7} \mid H_{0}^{*}\right)=\operatorname{Pr}\left(\chi^{2}(1)>t_{7}\right) .
$$

\section{SIMULATION STUDIES}

To investigate the performance of the likelihood ratio test (LRT) and the score test, we compare the type I error rate and the power of the two tests for (1) $\phi_{1}=0,(2) \phi_{0}=0$ and (3) $\lambda=\lambda_{0}$. And the sample sizes are set to be $n=$ $50(50) 500$.

\subsection{Tests for one inflation}

In this subsection, we compare the type I error rates (with $H_{0}: \phi_{1}=0$ ) and powers (with $H_{1}: \phi_{1}>0$ ) between the LRT and the score test for various sample sizes via simulations, where the values of $\phi_{1}$ in $H_{1}$ are chosen to be $0.01,0.03,0.05,0.07,0.10,0.15$. For a given combination of $\left(n, \phi_{0}=0.5, \phi_{1}, \lambda=2\right)$, we first independently draw $\mathbf{z}_{1}^{(l)}, \ldots, \mathbf{z}_{n}^{(l)} \stackrel{\text { iid }}{\sim}$ Multinomial $\left(1 ; \phi_{0}, \phi_{1}, \phi_{2}\right)$ for $l=1, \ldots, L$, where $\mathbf{z}_{i}^{(l)}=\left(Z_{0 i}^{(l)}, Z_{1 i}^{(l)}, Z_{2 i}^{(l)}\right)^{\top}, i=1, \ldots, n$. And then we independently generate $X_{1}^{(l)}, \ldots, X_{n}^{(l)} \stackrel{\text { iid }}{\sim} \operatorname{Poisson}(\lambda)$. Finally, we set

$$
\begin{aligned}
Y_{i}^{(l)}=Z_{1 i}^{(l)}+Z_{2 i}^{(l)} \cdot X_{i}^{(l)}, & i=1, \ldots, n \\
& l=1, \ldots, L(L=1,000) .
\end{aligned}
$$

All hypothesis testings are conducted at significant level of $\alpha=0.05$. Let $r_{k}$ denote the number of rejecting the null hypothesis $H_{0}: \phi_{1}=0$ by the test statistics $T_{k}(k=2,3)$ given by (5.6) and (5.9), respectively. Hence, the actual significance level can be estimated by $r_{k} / L$ with $\phi_{1}=0$ and the power of the test statistic $T_{k}$ can be estimated by $r_{k} / L$ with $\phi_{1}>0$. We repeat the process of estimating each significance level and each power for 1,000 times, respectively, to obtain $\left\{\hat{\alpha}_{k}\right\}_{k=1}^{1000}$ and $\left\{1-\hat{\beta}_{k}\right\}_{k=1}^{1000}$. Then we use the mean, $100(\alpha / 2)$ and $100(1-\alpha / 2)$ percentiles of these values to estimate the empirical level/power and the corresponding $(1-\alpha) 100 \%$ upper and lower bounds.

Figure 1 shows the comparison of type I error rates between the LRT and the score test for various sample sizes, and the $95 \%$ CIs for the empirical significance level associated with the two tests. We can see that the score test has the correct size around $\alpha=0.05$, while the LRT can control its type I error rates in a lower level (around 0.045). As far as we know, the lower the type I error rate the better the test's performance, thus the LRT has a better performance in controlling its type I error rates around the pre-chosen nominal level than the score test. An interpretation is as follows: For testing $H_{0}: \phi_{1}=0$ and $H_{0}: \phi_{0}=0$ (corresponding to Figure 3), since the LRT considers both the null and alternative situations, it is more sensitive in distinguishing two models, which results in a lower type I error rate.

Figure 2 gives the comparison of powers between the LRT and the score test for different values of $\phi_{1}>0$. It is not difficult to find that the LRT is always more powerful than the score test. A possible interpretation is as follows: For testing $H_{0}: \phi_{1}=0$ and $H_{0}: \phi_{0}=0$ (corresponding to Figure 4) which are one-sided tests, the LRT should be more powerful than the score test in the sense that the LRT evaluates things under both the null and alternative while the score test only evaluates things under the null, thus it is blind to the sign of the $\phi$. Then the score test is more likely to make type II error rates and is less powerful.

The empirical levels/powers of the LRT statistic $T_{2}$ and the score test statistic $T_{3}$ are summarized in Tables 1 and 2 , respectively, for six scenarios: $\phi_{1}=0.01,0.03,0.05,0.07$, $0.10,0.15$.

\subsection{Tests for zero inflation}

In this subsection, we compare the type I error rates (with $H_{0}: \phi_{0}=0$ ) and powers (with $H_{1}: \phi_{0}>0$ ) between the LRT and the score test for various sample sizes via simulations, where the values of $\phi_{0}$ in $H_{1}$ are set to be $0.01,0.03,0.05,0.07,0.10,0.15$. For a given combination 


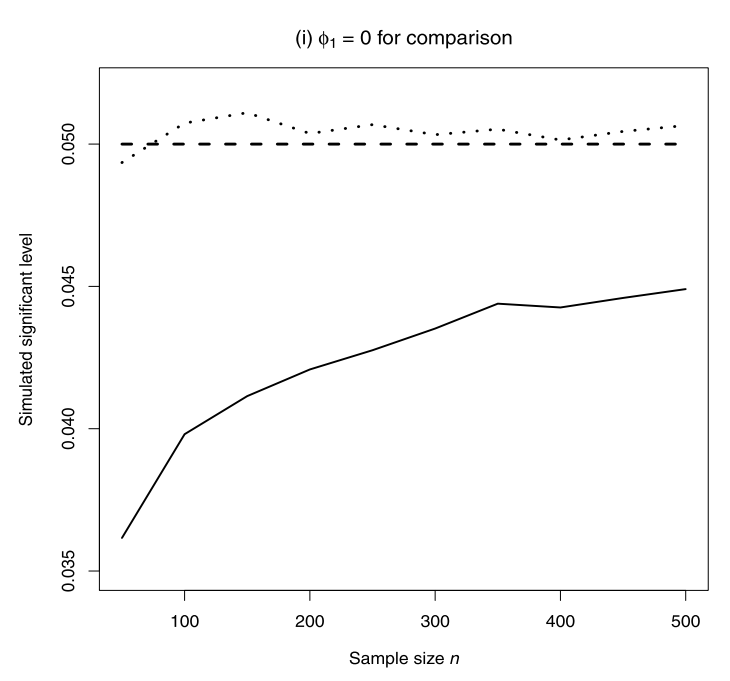

(a) $\phi_{1}=0.01$

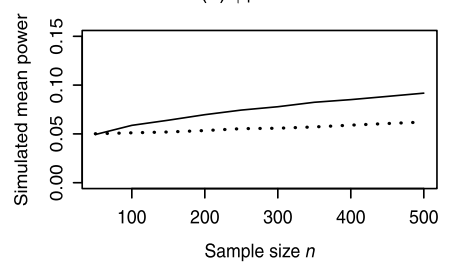

(c) $\phi_{1}=0.05$

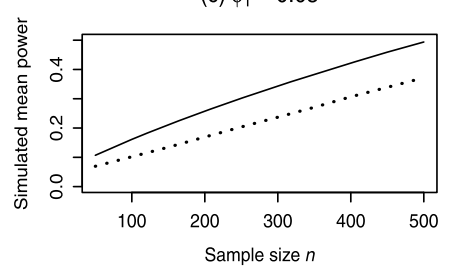

(e) $\phi_{1}=0.10$

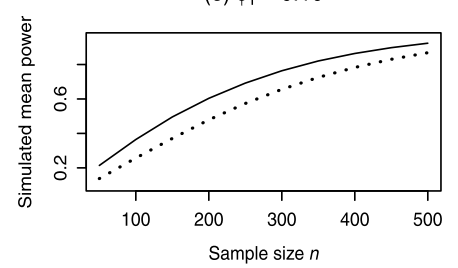

(b) $\phi_{1}=0.03$

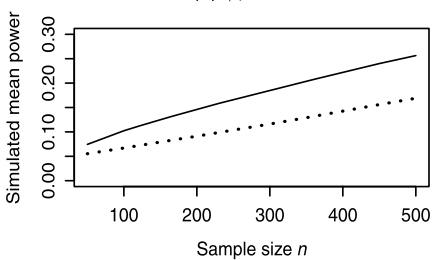

(d) $\phi_{1}=0.07$

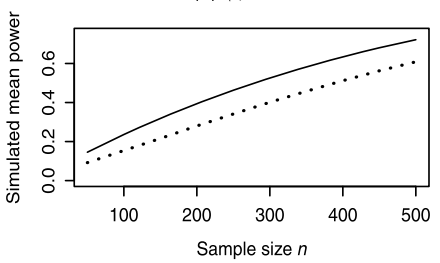

(f) $\phi_{1}=0.15$

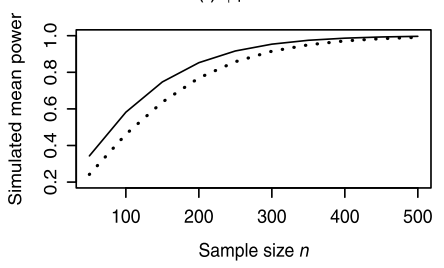

Figure 2. Comparison of powers between the LRT (solid line) and the score test (dotted line) for testing one inflation in the ZOIP model with $H_{0}: \phi_{1}=0$ against $H_{1}: \phi_{1}>0$ for different values of $\phi_{1}$.

Table 1. Empirical levels/powers of the LRT statistic $T_{2}$ based on 1,000 replications for $\phi_{0}=0.5$ and $\lambda=2$

\begin{tabular}{l|c|cccccc}
\hline \hline \multirow{2}{*}{$\begin{array}{l}\text { Sample } \\
\text { size }\end{array}$} & \multirow{2}{*}{$\begin{array}{c}\text { Empirical } \\
\text { level }\end{array}$} & \multicolumn{6}{|c}{ Empirical power } \\
\cline { 3 - 8 } & & \multicolumn{5}{|c}{$\phi_{1}$} \\
\cline { 3 - 8 } & & 0.010 & 0.030 & 0.050 & 0.070 & 0.100 & 0.150 \\
\hline 50 & 0.036 & 0.049 & 0.074 & 0.107 & 0.145 & 0.214 & 0.343 \\
100 & 0.040 & 0.059 & 0.102 & 0.161 & 0.236 & 0.365 & 0.582 \\
150 & 0.041 & 0.064 & 0.125 & 0.211 & 0.319 & 0.496 & 0.747 \\
200 & 0.042 & 0.070 & 0.147 & 0.257 & 0.394 & 0.604 & 0.852 \\
250 & 0.043 & 0.074 & 0.166 & 0.302 & 0.463 & 0.692 & 0.917 \\
300 & 0.044 & 0.078 & 0.184 & 0.343 & 0.525 & 0.764 & 0.954 \\
350 & 0.044 & 0.082 & 0.204 & 0.384 & 0.582 & 0.821 & 0.975 \\
400 & 0.044 & 0.085 & 0.222 & 0.422 & 0.634 & 0.864 & 0.986 \\
450 & 0.045 & 0.088 & 0.240 & 0.459 & 0.680 & 0.898 & 0.993 \\
500 & 0.045 & 0.092 & 0.256 & 0.494 & 0.722 & 0.924 & 0.996 \\
\hline
\end{tabular}

of $\left(n, \phi_{0}, \phi_{1}=0.5, \lambda=2\right)$, we generate $Y_{1}^{(l)}, \ldots, Y_{n}^{(l)} \stackrel{\mathrm{iid}}{\sim}$ $\operatorname{ZOIP}\left(\phi_{0}, 0.5 ; 2\right)$ with $L=1,000$ replications. All hypothesis testings are conducted at significant level of $\alpha=0.05$.

Let $r_{k}$ denote the number of rejecting the null hypothesis $H_{0}: \phi_{0}=0$ by the test statistics $T_{k}(k=4,5)$ given by $(5.12)$ and (5.15), respectively. Hence, the actual significance level can be estimated by $r_{k} / L$ with $\phi_{0}=0$ and the power of the test statistic $T_{k}$ can be estimated by $r_{k} / L$ with $\phi_{0}>0$. The estimated level/power and 95\% CIs are calculated in a similar way as in Section 6.1. 
Table 2. Empirical levels/powers of the score statistic $T_{3}$ based on 1,000 replications for $\phi_{0}=0.5$ and $\lambda=2$

\begin{tabular}{l|c|cccccc}
\hline \hline \multirow{2}{*}{$\begin{array}{l}\text { Sample } \\
\text { size }\end{array}$} & \multirow{2}{*}{$\begin{array}{c}\text { Empirical } \\
(n)\end{array}$} & \multicolumn{6}{|c}{ level } \\
\cline { 3 - 8 } & & \multicolumn{5}{|c}{$\phi_{1}$} \\
\cline { 3 - 8 } & & 0.010 & 0.030 & 0.050 & 0.070 & 0.100 & 0.150 \\
\hline 50 & 0.050 & 0.050 & 0.055 & 0.070 & 0.092 & 0.138 & 0.242 \\
100 & 0.051 & 0.051 & 0.067 & 0.102 & 0.154 & 0.257 & 0.461 \\
150 & 0.051 & 0.052 & 0.079 & 0.135 & 0.216 & 0.371 & 0.637 \\
200 & 0.050 & 0.053 & 0.091 & 0.169 & 0.280 & 0.479 & 0.769 \\
250 & 0.051 & 0.055 & 0.104 & 0.204 & 0.341 & 0.574 & 0.858 \\
300 & 0.050 & 0.056 & 0.116 & 0.237 & 0.401 & 0.656 & 0.915 \\
350 & 0.051 & 0.057 & 0.129 & 0.271 & 0.458 & 0.725 & 0.950 \\
400 & 0.050 & 0.059 & 0.143 & 0.307 & 0.512 & 0.783 & 0.972 \\
450 & 0.050 & 0.061 & 0.156 & 0.339 & 0.563 & 0.830 & 0.984 \\
500 & 0.051 & 0.062 & 0.169 & 0.372 & 0.609 & 0.869 & 0.991 \\
\hline
\end{tabular}

Figure 3 shows the comparison of type I error rates between the LRT and the score test, and the $95 \%$ CIs for the empirical level. Both the LRT and the score test have the correct size around $\alpha=0.05$. However, the LRT test performs relatively better in controlling its type I error rates around the pre-chosen nominal level than the score test.

Figure 4 gives the comparison of powers between the LRT and the score test for different values of $\phi_{0}$. We found that the score test is always less powerful than the LRT.

The empirical levels/powers of the LRT statistic $T_{4}$ and the score test statistic $T_{5}$ are summarized in Tables 3 and 4, respectively, for six scenarios: $\phi_{0}=$ $0.01,0.03,0.05,0.07,0.10,0.15$.

\subsection{Tests for $\lambda=\lambda_{0}$}

In this subsection, we compare the type I error rates (with $H_{0}: \lambda=\lambda_{0}$ ) and powers (with $H_{1}: \lambda \neq \lambda_{0}$ ) between the LRT and the score test for various sample sizes, different values of $\lambda_{0}$ (set to be 2, 3, 5, 7 for the comparison of empirical levels) and $\lambda=1.5$ for the comparison of empirical powers. For a given combination of $\left(n, \phi_{0}=0.3, \phi_{1}=0.1, \lambda\right)$, we generate $Y_{1}^{(l)}, \ldots, Y_{n}^{(l)} \stackrel{\text { iid }}{\sim} \operatorname{ZOIP}\left(\phi_{0}, \phi_{1} ; \lambda\right)$ with $L=1,000$ replications.

Let $r_{k}$ denote the number of rejecting the null hypothesis $H_{0}: \lambda=\lambda_{0}$ by the test statistics $T_{k}(k=6,7)$ given by (5.18) and (5.21), respectively. Hence, the actual significance level can be estimated by $r_{k} / L$ with $\lambda=\lambda_{0}$ and the power of the test statistic $T_{k}$ can be estimated by $r_{k} / L$ with $\lambda \neq \lambda_{0}$. All hypothesis testings are conducted at significant level of $\alpha=0.05$. The estimated significance level/power and $95 \%$ CIs are calculated in a similar way as in Section 6.1.

Figure 5 shows that some comparison of type I error rates between the LRT and the score test. When $\lambda_{0}=2$ and the sample size is smaller than 200 , the score test has a better performance in controlling its type I error rates around the pre-chosen nominal level than the LRT, while they are interlaced as the sample size becomes larger. When $\lambda_{0} \geqslant 3$, it
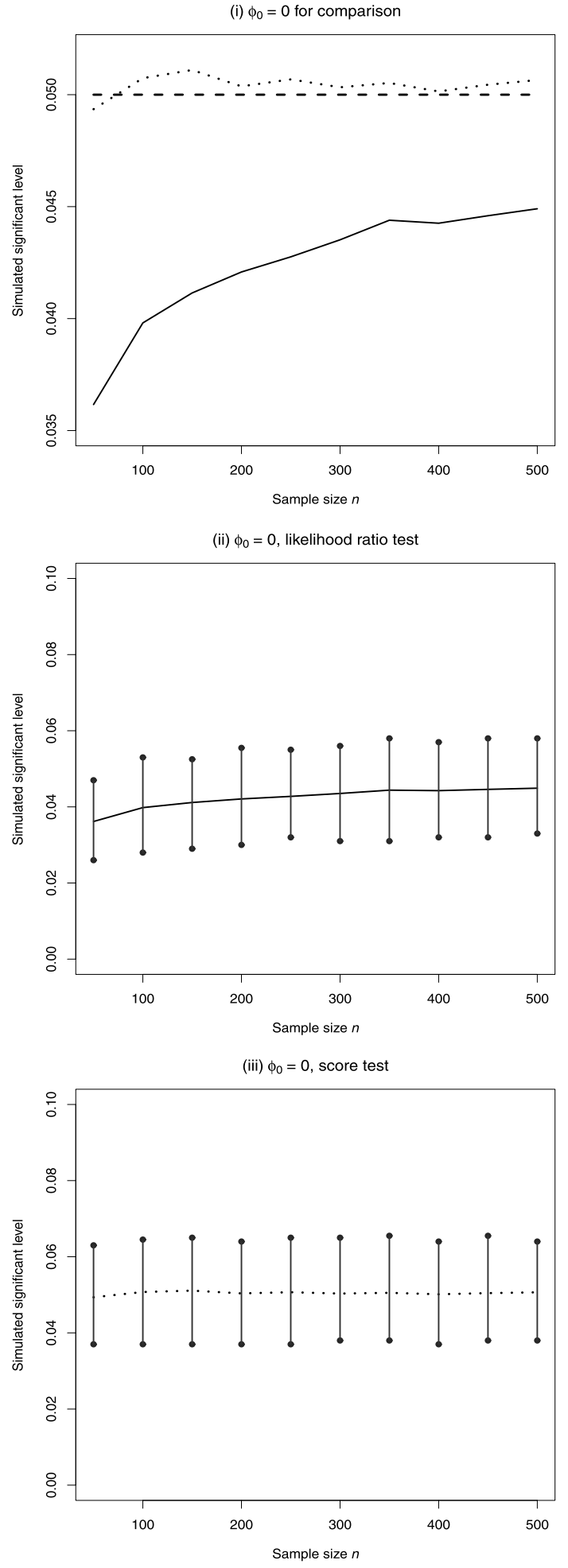

Figure 3. (i) Comparison of type I error rates between the LRT (solid line) and the score test (dotted line) for testing zero inflation in the ZOIP model with $H_{0}: \phi_{0}=0$ against $H_{1}$ : $\phi_{0}>0$. The dashed line is set as the predetermined significance level of $\alpha=0.05$; (ii) $95 \% \mathrm{Cls}$ for the empirical level of significance of the LRT; (iii) $95 \% \mathrm{Cls}$ for the empirical level of significance of the score test. 
(a) $\phi_{0}=0.01$

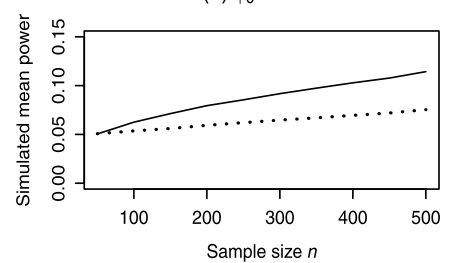

(c) $\phi_{0}=0.05$

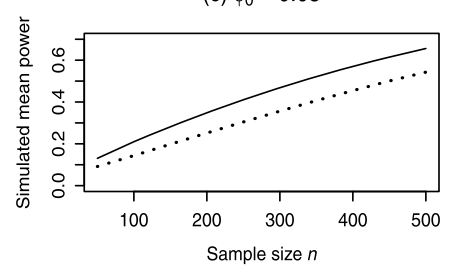

(e) $\phi_{0}=0.10$

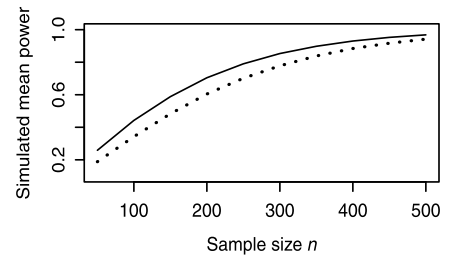

(b) $\phi_{0}=0.03$

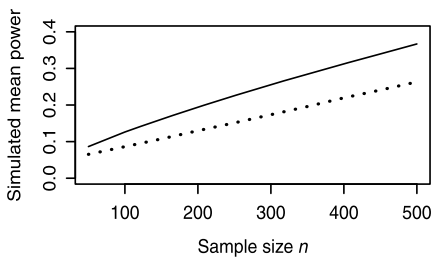

(d) $\phi_{0}=0.07$

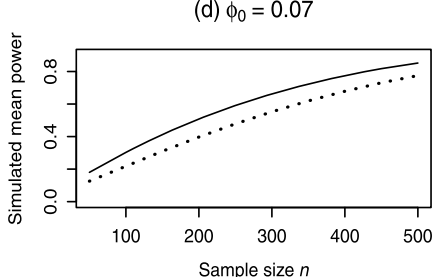

(f) $\phi_{0}=0.15$

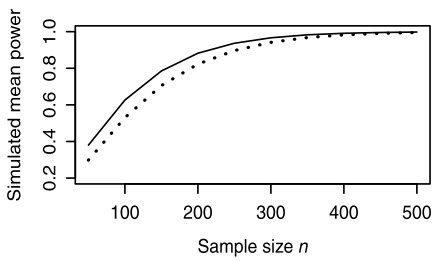

Figure 4. Comparison of powers between the LRT (solid line) and the score test (dotted line) for testing zero inflation in the ZOIP model with $H_{0}: \phi_{0}=0$ against $H_{1}: \phi_{0}>0$ for different values of $\phi_{0}$.

Table 3. Empirical levels/powers of the LRT statistic $T_{4}$ based on 1,000 replications for $\phi_{1}=0.5$ and $\lambda=2$

\begin{tabular}{|c|c|c|c|c|c|c|c|}
\hline \multirow{3}{*}{$\begin{array}{l}\text { Sample } \\
\text { size } \\
(n)\end{array}$} & \multirow{3}{*}{$\begin{array}{c}\text { Empirical } \\
\text { level }\end{array}$} & \multicolumn{6}{|c|}{ Empirical power } \\
\hline & & \multicolumn{6}{|c|}{$\phi_{0}$} \\
\hline & & 0.010 & 0.030 & 0.050 & 0.070 & 0.100 & 0.150 \\
\hline 50 & 0.036 & 0.050 & 0.086 & 0.131 & 0.180 & 0.258 & 0.380 \\
\hline 100 & 0.039 & 0.063 & 0.126 & 0.210 & 0.303 & 0.442 & 0.626 \\
\hline 150 & 0.041 & 0.071 & 0.161 & 0.281 & 0.412 & 0.588 & 0.785 \\
\hline 200 & 0.043 & 0.079 & 0.194 & 0.349 & 0.507 & 0.704 & 0.882 \\
\hline 250 & 0.043 & 0.085 & 0.224 & 0.410 & 0.591 & 0.790 & 0.937 \\
\hline 300 & 0.044 & 0.092 & 0.256 & 0.468 & 0.662 & 0.853 & 0.966 \\
\hline 350 & 0.044 & 0.097 & 0.284 & 0.521 & 0.723 & 0.898 & 0.983 \\
\hline 400 & 0.045 & 0.103 & 0.313 & 0.570 & 0.774 & 0.930 & 0.991 \\
\hline 450 & 0.044 & 0.108 & 0.340 & 0.615 & 0.818 & 0.953 & 0.996 \\
\hline 500 & 0.045 & 0.114 & 0.367 & 0.656 & 0.852 & 0.968 & 0.998 \\
\hline
\end{tabular}

can be seen that the two lines fluctuate with several points and sections, so we cannot say which one is more powerful. Thus when comparing the type I error rates between the two tests, there is no absolutely better one.

Figure 6 gives the comparison of powers between the LRT and the score test for the case with $\lambda=1.5 \neq \lambda_{0}=1$. It is not difficult to find that the LRT is slightly less powerful than the score test when sample size is less than 200 and the two tests almost have the same powers when the sample size is larger than 200 .

Table 4. Empirical levels/powers of the score statistic $T_{5}$ based on 1,000 replications for $\phi_{1}=0.5$ and $\lambda=2$

\begin{tabular}{|c|c|c|c|c|c|c|c|}
\hline \multirow{3}{*}{$\begin{array}{l}\text { Sample } \\
\text { size } \\
(n)\end{array}$} & \multirow{3}{*}{$\begin{array}{c}\text { Empirical } \\
\text { level }\end{array}$} & \multicolumn{6}{|c|}{ Empirical power } \\
\hline & & \multicolumn{6}{|c|}{$\phi_{0}$} \\
\hline & & 0.010 & 0.030 & 0.050 & 0.070 & 0.100 & $\overline{0.150}$ \\
\hline 50 & 0.049 & 0.051 & 0.065 & 0.092 & 0.126 & 0.188 & $\overline{0.298}$ \\
\hline 100 & 0.051 & 0.054 & 0.086 & 0.144 & 0.218 & 0.343 & 0.531 \\
\hline 150 & 0.050 & 0.056 & 0.107 & 0.198 & 0.310 & 0.483 & 0.706 \\
\hline 200 & 0.050 & 0.059 & 0.130 & 0.252 & 0.397 & 0.605 & 0.823 \\
\hline 250 & 0.050 & 0.062 & 0.151 & 0.305 & 0.478 & 0.702 & 0.897 \\
\hline 300 & 0.050 & 0.065 & 0.174 & 0.356 & 0.553 & 0.778 & 0.942 \\
\hline 350 & 0.051 & 0.067 & 0.196 & 0.407 & 0.620 & 0.838 & 0.968 \\
\hline 400 & 0.050 & 0.070 & 0.220 & 0.454 & 0.679 & 0.884 & 0.983 \\
\hline 450 & 0.050 & 0.072 & 0.241 & 0.501 & 0.731 & 0.917 & 0.991 \\
\hline 500 & 0.050 & 0.075 & 0.263 & 0.543 & 0.775 & 0.942 & 0.995 \\
\hline
\end{tabular}

(a) $\lambda_{0}=2$ for comparison

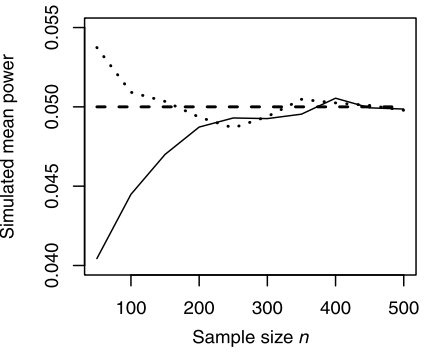

(c) $\lambda_{0}=5$ for comparison

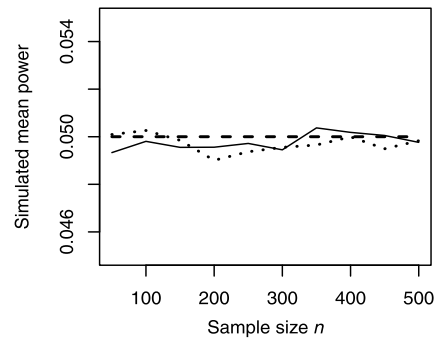

(b) $\lambda_{0}=3$ for comparison

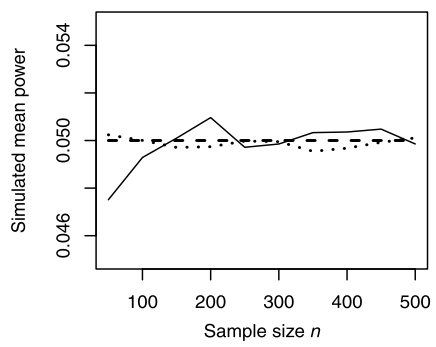

(d) $\lambda_{0}=7$ for comparison

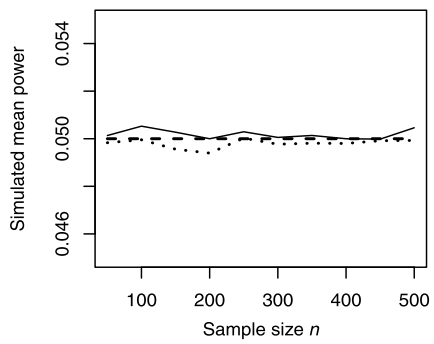

Figure 5. Comparison of type I error rates between the LRT (solid line) and the score test (dotted line) for testing $H_{0}$ : $\lambda=\lambda_{0}$ against $H_{1}: \lambda \neq \lambda_{0}$ with different values of $\lambda_{0}$. The dashed line is set as the predetermined significance level of

$$
\alpha=0.05 \text {. }
$$

The empirical levels/powers of the LRT statistic $T_{6}$ and the score test statistic $T_{7}$ are summarized in Tables 5 and 6 , respectively.

\section{APPLICATIONS}

In this section, five real data sets are used to illustrate the proposed methods, where the Newton-Raphson algorithm for finding the MLEs of parameters is not available in all examples because the corresponding observed information matrices are nearly singular, while the Fisher scoring and EM algorithms work well in all examples. 
Table 5. Empirical levels/powers of the LRT statistic $T_{6}$ based on 1,000 replications for $\phi_{0}=0.3$ and $\phi_{1}=0.1$

\begin{tabular}{l|cccc|c}
\hline \hline \multirow{2}{*}{$\begin{array}{l}\text { Sample size } \\
(n)\end{array}$} & \multicolumn{4}{|c|}{ Empirical level } & Empirical power \\
\cline { 2 - 6 } & $\lambda_{0}=2$ & $\lambda_{0}=3$ & $\lambda_{0}=5$ & $\lambda_{0}=7$ & $\lambda=1.5\left(H_{0}: \lambda=\lambda_{0}=1\right)$ \\
\hline 50 & 0.040 & 0.048 & 0.049 & 0.050 & 0.877 \\
100 & 0.044 & 0.049 & 0.050 & 0.051 & 0.987 \\
150 & 0.047 & 0.050 & 0.050 & 0.050 & 0.999 \\
200 & 0.049 & 0.051 & 0.050 & 0.050 & 1 \\
250 & 0.049 & 0.050 & 0.050 & 0.050 & 1 \\
300 & 0.049 & 0.050 & 0.049 & 0.050 & 1 \\
350 & 0.050 & 0.050 & 0.050 & 0.050 & 1 \\
400 & 0.051 & 0.050 & 0.050 & 0.050 & 1 \\
450 & 0.050 & 0.050 & 0.050 & 0.050 & 1 \\
500 & 0.050 & 0.050 & 0.050 & 0.050 & 1 \\
\hline
\end{tabular}

Table 6. Empirical levels/powers of the score statistic $T_{7}$ based on 1,000 replications for $\phi_{0}=0.3$ and $\phi_{1}=0.1$

\begin{tabular}{l|cccc|c}
\hline \hline \multirow{2}{*}{$\begin{array}{l}\text { Sample size } \\
(n)\end{array}$} & \multicolumn{4}{|c|}{ Empirical level } & Empirical power \\
\cline { 2 - 6 } & $\lambda_{0}=2$ & $\lambda_{0}=3$ & $\lambda_{0}=5$ & $\lambda_{0}=7$ & $\lambda=1.5\left(H_{0}: \lambda=\lambda_{0}=1\right)$ \\
\hline 50 & 0.054 & 0.050 & 0.050 & 0.050 & 0.968 \\
100 & 0.051 & 0.050 & 0.050 & 0.050 & 0.998 \\
150 & 0.050 & 0.050 & 0.050 & 0.050 & 1 \\
200 & 0.049 & 0.050 & 0.049 & 0.049 & 1 \\
250 & 0.049 & 0.050 & 0.049 & 0.050 & 1 \\
300 & 0.049 & 0.050 & 0.050 & 0.050 & 1 \\
350 & 0.050 & 0.050 & 0.050 & 0.050 & 1 \\
400 & 0.050 & 0.050 & 0.050 & 0.050 & 1 \\
450 & 0.050 & 0.050 & 0.049 & 0.050 & 1 \\
500 & 0.050 & 0.050 & 0.050 & 0.050 & 1 \\
\hline
\end{tabular}

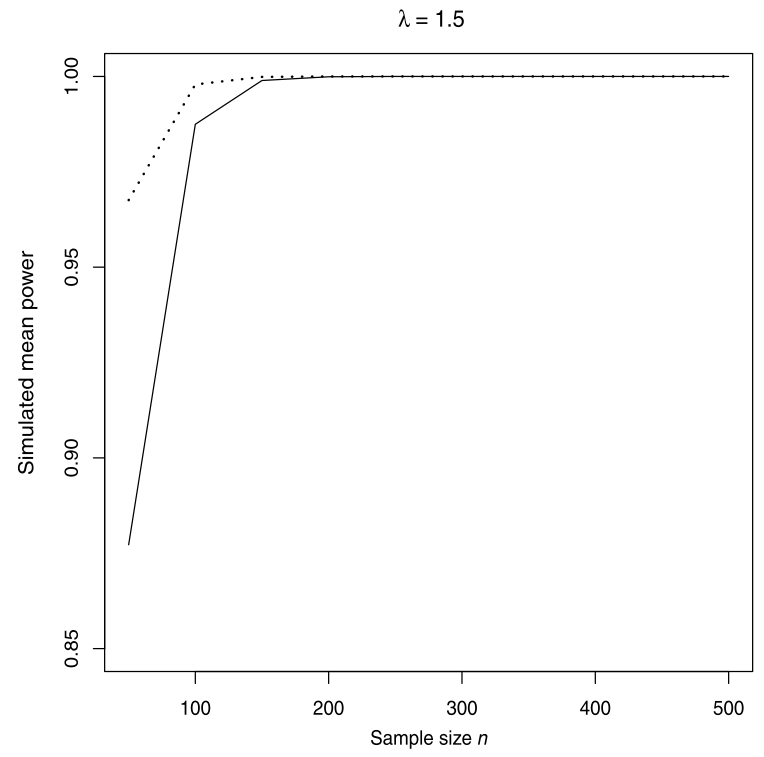

Figure 6. Comparison of powers between the LRT (solid line) and the score test (dotted line) for testing $H_{0}: \lambda=\lambda_{0}=1$ against $H_{1}: \lambda=1.5 \neq \lambda_{0}$.

\subsection{Dentist visiting data in Sweden}

Eriksson and Åberg [8] reported a two-year panel data from Swedish Level of Living Surveys in 1974 and 1991. To investigate the long term effect of the regular dentist visits during childhood and adolescence, the questions were set to know about people's habit of visiting dentist. The questions in the surveys are retrospective and in 1974 (1991) the questions refer to the individuals' situation in 1973 (1990). The panel includes 766 individuals who were between 15 and 29 years old in 1973. The number of visits to a dentist during the previous twelve month is only available for 1991, and whether the individual visits a dentist regularly is only available for 1974. Since we focus on modeling the distribution of visits to a dentist, we only gives the sample with different visit frequencies to a dentist in 1990 in Table 7.

\subsubsection{Likelihood-based inferences}

We noted that the data are characterized by both large proportions of zero visits and one visits to a dentist which are 17.5 and 41 percent, respectively. Therefore, the ZOIP distribution can be considered to capture the data. Let $Y_{1}, \ldots, Y_{n} \stackrel{\text { iid }}{\sim} \mathrm{ZOIP}\left(\phi_{0}, \phi_{1} ; \lambda\right)$. To find the MLEs of parameters, we choose $\left(\phi_{0}^{(0)}, \phi_{1}^{(0)}, \lambda^{(0)}\right)=(0.3,0.3,3)$ as their initial values. The MLEs of $\left(\phi_{0}, \phi_{1}, \lambda\right)$ converged to $\left(\hat{\phi}_{0}, \hat{\phi}_{1}, \hat{\lambda}\right)$ as shown in the second column of Table 8 in 3 iterations for 
Table 7. The dentist visiting data from Swedish Level of Living Surveys (Eriksson and Åberg, 1987; Melkersson and Olsson, 1999)

\begin{tabular}{l|cccccccccccccc}
\hline \hline Count & 0 & 1 & 2 & 3 & 4 & 5 & 6 & 7 & 8 & 9 & 10 & 12 & 15 & 20 \\
\hline Frequency & 134 & 314 & 149 & 69 & 32 & 26 & 14 & 6 & 1 & 0 & 11 & 3 & 3 & 4 \\
\hline
\end{tabular}

Table 8. MLEs and Cls of parameters for the dentist visiting data in Sweden

\begin{tabular}{l|cccccc}
\hline \hline Parameter & MLE & std $^{\mathrm{F}}$ & $95 \%$ asymptotic Wald CI & std $^{\mathrm{B}}$ & $95 \%$ bootstrap CI $^{\dagger}$ & $95 \%$ bootstrap CI $^{\ddagger}$ \\
\hline$\phi_{0}$ & 0.1535 & 0.0144 & {$[0.1253,0.1817]$} & 0.0146 & {$[0.1250,0.1821]$} & {$[0.1255,0.1828]$} \\
$\phi_{1}$ & 0.3422 & 0.0210 & {$[0.3010,0.3834]$} & 0.0212 & {$[0.3000,0.3831]$} & {$[0.3009,0.3828]$} \\
$\lambda$ & 3.1580 & 0.1169 & {$[2.9289,3.3870]$} & 0.1176 & {$[2.9272,3.3881]$} & {$[2.9340,3.3854]$} \\
\hline
\end{tabular}

$\operatorname{std}^{\mathrm{F}}$ : Square roots of the diagonal elements of inverse Fisher information matrix $J^{-1}\left(\phi_{0}, \phi_{1}, \lambda\right) \cdot$ std $^{\mathrm{B}}$ : Sample standard deviation of the bootstrap samples, cf. (4.11). CI $^{\dagger}$ : Normal-based bootstrap CI, cf. (4.12). CI ${ }^{\ddagger}$ : Non-normal-based bootstrap CI, cf. (4.13).

the Fisher scoring algorithm (4.5) and in 24 iterations for the EM algorithm (4.9)-(4.10), while the Newton-Raphson method is not available because the observed information matrix is nearly singular. The standard errors of the MLEs $\left(\hat{\phi}_{0}, \hat{\phi}_{1}, \hat{\lambda}\right)$ are given in the third column and $95 \%$ asymptotic Wald CIs (specified by (4.6)) of the three parameters are listed in the fourth column of Table 8 . With $G=6,000$ bootstrap replications, the two $95 \%$ bootstrap CIs of $\left(\phi_{0}, \phi_{1}, \lambda\right)$ are presented in the last two columns of Table 8.

Suppose we want to test $H_{0}:\left(\phi_{0}, \phi_{1}\right)=(0,0)$ against $H_{1}:\left(\phi_{0}, \phi_{1}\right) \neq(0,0)$. According to $(5.3)$, we calculate the value of the score test statistic which is given by $t_{1}=$ 217.3718 and from (5.4), we have $p_{\mathrm{v} 1}=0 \ll 0.05$. Thus, the $H_{0}$ should be rejected at the significance level of $\alpha=0.05$.

If we want to test the null hypothesis $H_{0}: \phi_{1}=0$ against $H_{1}: \phi_{1}>0$ at $\alpha=0.05$. According to (5.6) and (5.9), we calculate the values of the likelihood ratio test statistic and score test statistic, which are given by $t_{2}=214.6707$ and $t_{3}=214.0573$, respectively. Then, from (5.8) and (5.10), we have $p_{\mathrm{v} 2}=p_{\mathrm{v} 3}=0 \ll \alpha=0.05$, resulting in a rejection of $H_{0}$.

If we want to test $H_{0}: \phi_{0}=0$ against $H_{1}: \phi_{0}>0$. According to (5.12) and (5.15), we calculate the values of the likelihood ratio test statistic and score test statistic which are given by $t_{4}=146.3721$ and $t_{5}=161.5884$, respectively. Then, from (5.14) and (5.16), we have $p_{\mathrm{v} 4}=p_{\mathrm{v} 5}=0 \ll$ 0.05 . As a result, the $H_{0}$ should be rejected at the level of $\alpha=0.05$.

\subsubsection{Model comparison}

We assess the goodness-of-fit by Pearson's chi-squared test (Pearson, [23]) via the predicted counts and we also use the Akaike information criterion (AIC; Akaike, [1]) and Bayesian information criterion (BIC; Schwarz, [27]) to compare models. A comparison of the fitted Poisson, ZIP and ZOIP distributions is shown in Table 9. According to Pearson's goodness-of-fit criterion, although the values of Pearson's chi-squared statistics for the three fitted distributions are sharply decreasing from the highest 792.9705 to 638.0514
Table 9. Comparison of the fitted frequencies from Poisson, ZIP and ZOIP distribution for the dentist visiting data

\begin{tabular}{l|cccc}
\hline \hline Count & $\begin{array}{c}\text { Observed } \\
\text { frequency }\end{array}$ & Poisson & ZIP & ZOIP \\
\hline 0 & 134 & 110.66 & 134.00 & 134.00 \\
1 & 314 & 214.10 & 192.69 & 314.00 \\
2 & 149 & 207.11 & 196.55 & 81.88 \\
3 & 69 & 133.57 & 133.66 & 86.20 \\
4 & 32 & 64.60 & 68.17 & 68.05 \\
5 & 26 & 25.00 & 27.81 & 42.98 \\
6 & 14 & 8.06 & 9.46 & 22.62 \\
7 & 6 & 2.23 & 2.76 & 10.21 \\
$8-20$ & 22 & 0.68 & 0.90 & 6.06 \\
\hline$\phi_{0}$ & & & 0.0516 & 0.1535 \\
$\phi_{1}$ & & & & 0.3422 \\
$\lambda$ & & 1.9347 & 2.0400 & 3.1580 \\
\hline Pearson's $\chi^{2}$ & & 792.97 & 638.05 & 131.18 \\
d.f. & & 7 & 6 & 5 \\
$p$-value & & $<0.001$ & $<0.001$ & $<0.001$ \\
\hline AIC & & 3182.05 & 3175.78 & $\mathbf{2 9 6 3 . 1 1}$ \\
BIC & & 3186.70 & 3185.06 & $\mathbf{2 9 7 7 . 0 3}$ \\
\hline
\end{tabular}

Table 10. Criminal acts data (Dieckmann, 1981)

\begin{tabular}{l|llllll}
\hline \hline Count & 0 & 1 & 2 & 3 & 4 & 5 \\
\hline Frequency & 4037 & 219 & 29 & 9 & 5 & 2 \\
\hline
\end{tabular}

to the lowest 131.1833, all of the three distributions exhibit lack of fit to the data. However, the tests for testing three hypotheses $H_{0}:\left(\phi_{0}, \phi_{1}\right)=(0,0), H_{0}: \phi_{1}=0$ and $H_{0}: \phi_{0}=0$ reject all three $H_{0}$ 's at the significant level $\alpha=0.05$, indicating the existence of excess zeros and ones in the distribution. Moreover, both AIC and BIC criteria favor the ZOIP distribution.

\subsection{Criminal acts data}

Dieckmann [7] provided a data set from crime sociology consisting a sample of people with deviating behavior. Table 10 lists the distribution of number of criminal 
Table 11. MLEs and Cls of parameters for the criminal acts data

\begin{tabular}{l|cccccc}
\hline \hline Parameter & MLE & std $^{\mathrm{F}}$ & $95 \%$ asymptotic Wald CI & std $^{\mathrm{B}}$ & $95 \%$ bootstrap CI $^{\dagger}$ & $95 \%$ bootstrap CI $^{\ddagger}$ \\
\hline$\phi_{0}$ & 0.9316 & 0.0053 & {$[0.9212,0.9420]$} & 0.0064 & {$[0.9176,0.9428]$} & {$[0.9157,0.9405]$} \\
$\phi_{1}$ & 0.0415 & 0.0045 & {$[0.0326,0.0504]$} & 0.0048 & {$[0.0314,0.0504]$} & {$[0.0309,0.0498]$} \\
$\lambda$ & 1.3431 & 0.2447 & {$[0.8635,1.8227]$} & 0.2466 & {$[0.8537,1.8202]$} & {$[0.8501,1.8198]$} \\
\hline
\end{tabular}

$\operatorname{std}^{\mathrm{F}}:$ Square roots of the diagonal elements of inverse Fisher information matrix $J^{-1}\left(\phi_{0}, \phi_{1}, \lambda\right)$. std ${ }^{\mathrm{B}}$ : Sample standard deviation of the bootstrap samples, cf. (4.11). CI $^{\dagger}$ : Normal-based bootstrap CI, cf. (4.12). CI ${ }^{\ddagger}$ : Non-normal-based bootstrap CI, cf. (4.13).

acts of 4301 persons with deviating behavior. Dieckmann [7] pointed out that the Poisson model does not fit the data well. Böhning [2] showed that the fit of the ZIP model is still not good in the upper classes (i.e., number of criminal acts 3,4 , and 5). By observing that the data have relatively higher proportions of zeros and ones, while the frequencies for other categories are quite low, we apply the ZOIP model to fit the data.

\subsubsection{Likelihood-based inferences}

Data in Table 10 exhibit excess zeros and ones, while the traditional Poisson model does not adequately capture such characteristics, so we consider the ZOIP model. Let $Y_{1}, \ldots, Y_{n} \stackrel{\mathrm{iid}}{\sim} \operatorname{ZOIP}\left(\phi_{0}, \phi_{1} ; \lambda\right)$. To find the MLEs of $\left(\phi_{0}, \phi_{1}, \lambda\right)$, we choose $\left(\phi_{0}^{(0)}, \phi_{1}^{(0)}, \lambda^{(0)}\right)=(0.3,0.3,3)$ as the initial values. The MLEs of $\left(\phi_{0}, \phi_{1}, \lambda\right)$ converged to $\left(\hat{\phi}_{0}, \hat{\phi}_{1}, \hat{\lambda}\right)$ as shown in the second column of Table 11 in 6 iterations for the Fisher scoring algorithm (4.5) and in 249 iterations for the EM algorithm (4.9)-(4.10). The standard errors of the MLEs $\left(\hat{\phi}_{0}, \hat{\phi}_{1}, \hat{\lambda}\right)$ are given in the third column and $95 \%$ asymptotic Wald CIs (i.e. (4.6)) of the three parameters are listed in the fourth column of Table 11. With $G=6,000$ bootstrap replications, the two $95 \%$ bootstrap CIs of $\left(\phi_{0}, \phi_{1}, \lambda\right)$ are shown in the last two columns of Table 11.

Let $\alpha=0.05$. Suppose we want to test $H_{0}:\left(\phi_{0}, \phi_{1}\right)=$ $(0,0)$ against $H_{1}:\left(\phi_{0}, \phi_{1}\right) \neq(0,0)$. According to $(5.3)$, we calculate the value of the score test statistic which is given by $t_{1}=1848.2450$ and from $(5.4)$, we have $p_{\mathrm{v} 1}=0 \ll 0.05$. Thus, the $H_{0}$ should be rejected at the significance level of $\alpha=0.05$.

Suppose we want to test the null hypothesis $H_{0}: \phi_{1}=0$ against the alternative hypothesis $H_{1}: \phi_{1}>0$. According to (5.6) and (5.9), we calculate the values of the likelihood ratio test and score test statistics, which are given by $t_{2}=25.5011$ and $t_{3}=30.0044$, respectively. Then from (5.8) and (5.10), we have $p_{\mathrm{v} 2}=p_{\mathrm{v} 3}=0 \ll \alpha$. Thus, we should reject $H_{0}$.

\subsubsection{Model comparison}

We assess the model fitting by Pearson's chi-squared test, $\mathrm{AIC}$ and BIC. We list the fitted frequencies from Poisson, ZIP and ZOIP models in Table 12 and compute the corresponding $p$-values and MLEs of parameters. According to Pearson's goodness-of-fit criterion, the Poisson and ZIP
Table 12. Comparison of the fitted frequencies from Poisson, ZIP and ZOIP distributions for the criminal acts

data

\begin{tabular}{l|cccc}
\hline \hline Count & $\begin{array}{c}\text { Observed } \\
\text { frequency }\end{array}$ & Poisson & ZIP & ZOIP \\
\hline 0 & 4037 & 3979.64 & 4037.00 & 4037.00 \\
1 & 219 & 309.04 & 204.54 & 219.00 \\
2 & 29 & 12.00 & 50.15 & 27.28 \\
3 & 9 & 0.31 & 8.20 & 12.21 \\
4 & 5 & 0.01 & 1.01 & 4.10 \\
5 & 2 & 0.00 & 0.12 & 1.40 \\
\hline Pearson's $\chi^{2}$ & & 46582.53 & 59.31 & 1.40 \\
d.f. & & 4 & 3 & 2 \\
$p$-value & & $<0.001$ & $<0.001$ & 0.4958 \\
\hline $4+$ & 7 & 0.01 & 1.11 & 5.51 \\
Pearson's $\chi^{2}$ & & 8279.97 & 41.19 & 1.36 \\
d.f. & & 3 & 2 & 1 \\
$p$-value & & $<0.001$ & $<0.001$ & 0.2436 \\
\hline$\phi_{0}$ & & & 0.8416 & 0.9316 \\
$\phi_{1}$ & & 0.0777 & 0.4904 & 1.3431 \\
$\lambda$ & & 2500.43 & 2346.80 & $\mathbf{2 3 2 3 . 3 0}$ \\
\hline AIC & & 2506.80 & 2359.54 & $\mathbf{2 3 4 2 . 4 0}$ \\
BIC & & & & \\
\hline
\end{tabular}

models exhibit lack of fit to the data because of very small $p$-values while the ZOIP model shows a satisfactory fit. Furthermore, both the likelihood ratio test and the score test support the ZOIP model. Finally, both AIC and BIC favor the ZOIP model, so the ZOIP model is more favorable than the other two models.

\subsection{Fetal lamb movement data}

Leroux and Puterman [17] analyzed one particular sequence of counts in a study of breathing and body movements in fetal lambs designed to examine the possible changes in the amount and pattern of fetal activity during the last two-thirds of gestation. Table 13 lists the number of movements made by a fetal lamb observed through ultrasound in 240 consecutive 5 -second intervals. Gupta et al. [11] further analyzed this data set by the zero-adjusted generalized Poisson distribution which results in a better fit than both the traditional Poisson distribution and a mixture of two Poisson distributions. Alternatively, we can apply the ZOIP distribution to fit this data set since there are extra zeros and ones. 
Table 13. Number of movements made by a fetal lamb (Leroux and Puterman, 1992) and fitted frequencies from ZIP and ZOIP distributions

\begin{tabular}{l|cccccccc}
\hline \hline Number of movements & 0 & 1 & 2 & 3 & 4 & 5 & 6 & 7 \\
\hline Number of intervals & 182 & 41 & 12 & 2 & 2 & 0 & 0 & 1 \\
Fitted frequencies (ZIP) & 182.00 & 36.86 & 15.61 & 4.41 & 0.93 & 0.16 & 0.02 & 0.00 \\
Fitted frequencies (ZOIP) & 182.00 & 41.00 & 9.56 & 4.85 & 1.85 & 0.56 & 0.14 & 0.04 \\
\hline
\end{tabular}

Table 14. MLEs and Cls of parameters for the fetal lamb movement data

\begin{tabular}{l|ccccrr}
\hline \hline Parameter & MLE & std $^{\mathrm{F}}$ & $95 \%$ asymptotic Wald CI & std $^{\mathrm{B}}$ & $95 \%$ bootstrap CI $^{\dagger}$ & $95 \%$ bootstrap CI $^{\ddagger}$ \\
\hline$\phi_{0}$ & 0.7240 & 0.0407 & {$[0.6442,0.8038]$} & 0.0570 & {$[0.5965,0.8198]$} & {$[0.5539,0.7891]$} \\
$\phi_{1}$ & 0.1185 & 0.0369 & {$[0.0461,0.1909]$} & 0.0408 & {$[0.0297,0.1897]$} & {$[0.0099,0.1820]$} \\
$\lambda$ & 1.5224 & 0.4142 & {$[0.7106,2.3342]$} & 0.4150 & {$[0.6955,2.3224]$} & {$[0.7289,2.3517]$} \\
\hline
\end{tabular}

$\operatorname{std}^{\mathrm{F}}$ : Square roots of the diagonal elements of inverse Fisher information matrix $J^{-1}\left(\phi_{0}, \phi_{1}, \lambda\right)$. std ${ }^{\mathrm{B}}$ : Sample standard deviation of the bootstrap samples, cf. (4.11). $\mathrm{CI}^{\dagger}$ : Normal-based bootstrap CI, cf. (4.12). CI ${ }^{\ddagger}$ : Non-normal-based bootstrap CI, cf. (4.13).

\subsubsection{Likelihood-based inferences}

To find the MLEs of $\left(\phi_{0}, \phi_{1}, \lambda\right)$, we choose $\left(\phi_{0}^{(0)}, \phi_{1}^{(0)}\right.$, $\left.\lambda^{(0)}\right)=(0.3,0.3,3)$ as the initial values. The MLEs of $\left(\phi_{0}, \phi_{1}, \lambda\right)$ converged to $\left(\hat{\phi}_{0}, \hat{\phi}_{1}, \hat{\lambda}\right)$ as shown in the second column of Table 14 in 6 iterations for the Fisher scoring algorithm (4.5) and in 131 iterations for EM algorithm (4.9)(4.10). The standard errors of the MLEs $\left(\hat{\phi}_{0}, \hat{\phi}_{1}, \hat{\lambda}\right)$ are given in the third column and $95 \%$ asymptotic Wald CIs (i.e. (4.6)) of the three parameters are listed in the fourth column of Table 14. With $G=6,000$ bootstrap replications, the two $95 \%$ bootstrap CIs of $\left(\phi_{0}, \phi_{1}, \lambda\right)$ are reported in the last two columns of Table 14 .

Let $\alpha=0.05$. Suppose we want to test $H_{0}:\left(\phi_{0}, \phi_{1}\right)=$ $(0,0)$ against $H_{1}:\left(\phi_{0}, \phi_{1}\right) \neq(0,0)$. According to $(5.3)$, we calculate the value of the score test statistic which is given by $t_{1}=57.0687$ and from $(5.4)$, we have $p_{\mathrm{v} 1}=0 \ll 0.05$. Thus, the $H_{0}$ should be rejected at the significance level of $\alpha=0.05$.

Suppose we want to test the null hypothesis $H_{0}: \phi_{1}=0$ against the alternative hypothesis $H_{1}: \phi_{1}>0$. According to (5.6) and (5.9), we calculate the values of the likelihood ratio test statistic and score test statistic, which are given by $t_{2}=4.9434$ and $t_{3}=5.1433$, respectively. Then from (5.8) and (5.10), we have $p_{\mathrm{v} 2}=0.0131$ and $p_{\mathrm{v} 3}=0.0233$, both are less than 0.05 . Thus, we should reject $H_{0}$.

\subsubsection{Model comparison}

The model fitting is conducted by Pearson's chi-squared test, AIC and BIC. The corresponding results are listed in Table 15. According to Pearson's chi-squared test statistics, the ZIP model does not exhibit lack of fit, but the ZOIP model substantially improves the fit. In Section 7.3.1, we have shown that the tests for testing $H_{0}:\left(\phi_{0}, \phi_{1}\right)=(0,0)$ and $H_{0}: \phi_{1}=0$ reject the $H_{0}$ at the significant level of $\alpha=0.05$, indicating the existence of excess zeros and ones in the model. The AIC favors the ZOIP model, while the BIC favors the ZIP model. From Table 13, we can see that the number of zero counts and the number of one counts
Table 15. Results of the model fitting for the fetal lamb movement data

\begin{tabular}{l|cc}
\hline \hline & ZIP & ZOIP \\
\hline $4+$ & & \\
Pearson's $\chi^{2}$ & 5.79 & 2.36 \\
d.f. & 2 & 1 \\
$p$-value & 0.0553 & 0.1242 \\
\hline $5+$ & & \\
Pearson's $\chi^{2}$ & 7.46 & 2.40 \\
d.f. & 3 & 2 \\
$p$-value & 0.0585 & 0.3011 \\
\hline$\phi_{0}$ & 0.5771 & 0.7240 \\
$\phi_{1}$ & & 0.1185 \\
$\lambda$ & 0.8473 & 1.5224 \\
\hline AIC & 384.87 & $\mathbf{3 8 1 . 9 3}$ \\
BIC & $\mathbf{3 9 1 . 8 4}$ & 392.37 \\
\hline & & \\
\end{tabular}

predicted by the ZOIP model are close to the corresponding observed counts, and the ZOIP model fits better than the ZIP model for most of other count categories. Therefore, the ZOIP model is a more reasonable alternative model.

\subsection{Death notice data of London Times}

The data are the numbers of death notices of women 80 years of age and over, appearing in the London Times on each day for three consecutive years (Schilling, [26]). The data set was fitted by Hasselblad [12] with a mixture of two Poisson distributions. Subsequently, Gupta et al. [11] analyzed the data by the zero-adjusted generalized Poisson distribution with smaller variances of estimates when comparing with the mixture model. The counts are given in Table 16. Considering the relatively higher proportions of zero and one counts, we apply the ZOIP distribution to fit the data.

\subsubsection{Likelihood-based inferences}

To find the MLEs of parameters, we choose $\left(\phi_{0}^{(0)}, \phi_{1}^{(0)}\right.$, $\left.\lambda^{(0)}\right)=(0.3,0.3,3)$ as the initial values. The MLEs of 
Table 16. Death notice data of London Times (Schilling, 1947)

\begin{tabular}{l|cccccccccc}
\hline \hline Observed death count & 0 & 1 & 2 & 3 & 4 & 5 & 6 & 7 & 8 & 9 \\
\hline Frequency & 162 & 267 & 271 & 185 & 111 & 61 & 27 & 8 & 3 & 1 \\
\hline
\end{tabular}

Table 17. MLEs and Cls of parameters for the death notice data of London Times

\begin{tabular}{l|cccccc}
\hline \hline Parameter & MLE & std $^{\mathrm{F}}$ & $95 \%$ asymptotic Wald CI & std $^{\mathrm{B}}$ & $95 \%$ bootstrap CI $^{\dagger}$ & $95 \%$ bootstrap CI $^{\ddagger}$ \\
\hline$\phi_{0}$ & 0.0660 & 0.0144 & {$[0.0379,0.0942]$} & 0.0143 & {$[0.0375,0.0936]$} & {$[0.0371,0.0931]$} \\
$\phi_{1}$ & 0.0488 & 0.0212 & {$[0.0072,0.0904]$} & 0.0207 & {$[0.0074,0.0885]$} & {$[0.0062,0.0874]$} \\
$\lambda$ & 2.3816 & 0.0751 & {$[2.2345,2.5287]$} & 0.0739 & {$[2.2356,2.5252]$} & {$[2.2354,2.5231]$} \\
\hline
\end{tabular}

$\operatorname{std}^{\mathrm{F}}$ : Square roots of the diagonal elements of inverse Fisher information matrix $J^{-1}\left(\phi_{0}, \phi_{1}, \lambda\right)$. std ${ }^{\mathrm{B}}$ : Sample standard deviation of the bootstrap samples, cf. (4.11). CI $^{\dagger}$ : Normal-based bootstrap CI, cf. (4.12). CI ${ }^{\ddagger}$ : Non-normal-based bootstrap CI, cf. (4.13).

$\left(\phi_{0}, \phi_{1}, \lambda\right)$ converged to $\left(\hat{\phi}_{0}, \hat{\phi}_{1}, \hat{\lambda}\right)$ as shown in the second column of Table 17 in 5 iterations for the Fisher scoring algorithm (4.5) and in 200 iterations for the EM algorithm (4.9)-(4.10). The standard errors of the MLEs $\left(\hat{\phi}_{0}, \hat{\phi}_{1}, \hat{\lambda}\right)$ are given in the third column and $95 \%$ asymptotic Wald CIs (i.e. (4.6)) of the three parameters are listed in the fourth column of Table 17. With $G=6,000$ bootstrap replications, the two $95 \%$ bootstrap CIs of $\left(\phi_{0}, \phi_{1}, \lambda\right)$ are provided in the last two columns of Table 17.

Let $\alpha=0.05$. Suppose we want to test $H_{0}:\left(\phi_{0}, \phi_{1}\right)=$ $(0,0)$ against $H_{1}:\left(\phi_{0}, \phi_{1}\right) \neq(0,0)$. According to $(5.3)$, we calculate the value of the score test statistic which is given by $t_{1}=20.6166$ and from (5.4), we have $p_{\mathrm{v} 1}=0 \ll 0.05$. Thus, the $H_{0}$ should be rejected at the significance level of $\alpha=0.05$.

Suppose we want to test $H_{0}: \phi_{1}=0$ against $H_{1}: \phi_{1}>0$. According to (5.6) and (5.9), we calculate the values of the LRT statistic and score test statistic, which are given by $t_{2}=5.0760$ and $t_{3}=5.1068$, respectively. Then, from (5.8) and (5.10), we have $p_{\mathrm{v} 2}=0.0121$ and $p_{\mathrm{v} 3}=0.0238$, both are less than 0.05 . Thus we should reject $H_{0}$.

\subsubsection{Model comparison}

The model fitting is conducted by Pearson's chi-squared test, AIC and BIC. We listed the predicted frequencies in Table 18 to compare Poisson, ZIP and ZOIP models. The computed Pearson's chi-square statistics indicate that the Poisson model exhibits lack of fit, the ZIP model does not exhibit lack of fit, but the ZOIP model substantially improves the fit. Moreover, both the LRT and the score test favor the ZOIP model. However, the AIC favors the ZOIP model, while the BIC favors the ZIP model.

\subsection{Ammunition factory accidents data}

Table 19 lists the number of accidents of 647 female workers in an ammunition factory (Greenwood and Yule, [10]). Since the simple Poisson model gave a bad fit to the data, Böhning [2] fitted the data by the ZIP model. From the result of Pearson's chi-squared test from Böhning [2], it seems that the ZIP model still has a lack of fit to the data, so we will apply the ZOIP model.
Table 18. Comparison of the fitted frequencies from Poisson, ZIP and ZOIP distribution for the death notice data of London Times

\begin{tabular}{l|cccc}
\hline \hline Count & $\begin{array}{c}\text { Observed } \\
\text { frequency }\end{array}$ & Poisson & ZIP & ZOIP \\
\hline 0 & 162 & 126.78 & 162.00 & 162.00 \\
1 & 267 & 273.47 & 244.38 & 267.00 \\
2 & 271 & 294.92 & 277.29 & 254.22 \\
3 & 185 & 212.04 & 209.76 & 201.82 \\
4 & 111 & 114.34 & 119.01 & 120.17 \\
5 & 61 & 49.33 & 54.02 & 57.24 \\
6 & 27 & 17.73 & 20.43 & 22.72 \\
7 & 8 & 5.46 & 6.62 & 7.73 \\
8 & 3 & 1.47 & 1.88 & 2.30 \\
9 & 1 & 0.45 & 0.61 & 0.79 \\
\hline Pearson's $\chi^{2}$ & & 26.47 & 9.92 & 4.54 \\
d.f. & & 8 & 7 & 6 \\
$p$-value & & 0.0009 & 0.1931 & 0.6044 \\
\hline $7+$ & 12 & 7.38 & 9.11 & 10.82 \\
Pearson's $\chi^{2}$ & & 25.91 & 9.63 & 4.39 \\
d.f. & & 6 & 5 & 4 \\
$p$-value & & 0.0002 & 0.0864 & 0.3558 \\
\hline$\phi_{0}$ & & & 0.0496 & 0.0660 \\
$\phi_{1}$ & & & & 0.0488 \\
$\lambda$ & & 2.1569 & 2.2694 & 2.3816 \\
\hline AIC & & 4004.80 & 3992.10 & $\mathbf{3 9 8 9 . 0 3}$ \\
BIC & & 4009.80 & $\mathbf{4 0 0 2 . 1 0}$ & 4004.03 \\
\hline
\end{tabular}

Table 19. Ammunition factory accidents data (Greenwood and Yule, 1920)

\begin{tabular}{l|llllll}
\hline \hline Count & 0 & 1 & 2 & 3 & 4 & 5 \\
\hline Frequency & 447 & 132 & 42 & 21 & 3 & 2 \\
\hline
\end{tabular}

\subsubsection{Likelihood-based inferences}

To find the MLEs of parameters, we choose $\left(\phi_{0}^{(0)}, \phi_{1}^{(0)}\right.$, $\left.\lambda^{(0)}\right)=(0.3,0.3,3)$ as the initial values. The MLEs of $\left(\phi_{0}, \phi_{1}, \lambda\right)$ converged to $\left(\hat{\phi}_{0}, \hat{\phi}_{1}, \hat{\lambda}\right)$ as shown in the second column of Table 20 in 6 iterations for the Fisher scoring 
Table 20. MLEs and Cls of parameters for the ammunition factory accidents data

\begin{tabular}{l|cccccc}
\hline \hline Parameter & MLE & std $^{\mathrm{F}}$ & $95 \%$ asymptotic Wald CI & std $^{\mathrm{B}}$ & $95 \%$ bootstrap CI $^{\dagger}$ & $95 \%$ bootstrap CI $^{\ddagger}$ \\
\hline$\phi_{0}$ & 0.5969 & 0.0452 & {$[0.5084,0.6855]$} & 0.0515 & {$[0.4844,0.6862]$} & {$[0.4639,0.6647]$} \\
$\phi_{1}$ & 0.0913 & 0.0347 & {$[0.0233,0.1594]$} & 0.0359 & {$[0.0156,0.1565]$} & {$[0.0036,0.1501]$} \\
$\lambda$ & 1.1994 & 0.1918 & {$[0.8236,1.5752]$} & 0.1915 & {$[0.8196,1.5704]$} & {$[0.0036,0.1501]$} \\
\hline
\end{tabular}

$\mathrm{std}^{\mathrm{F}}$ : Square roots of the diagonal elements of inverse Fisher information matrix $J^{-1}\left(\phi_{0}, \phi_{1}, \lambda\right)$. std ${ }^{\mathrm{B}}$ : Sample standard deviation of the bootstrap samples, cf. (4.11). CI $^{\dagger}$ : Normal-based bootstrap CI, cf. (4.12). CI ${ }^{\ddagger}$ : Non-normal-based bootstrap CI, cf. (4.13).

algorithm (4.5) and in 412 iterations for the EM algorithm (4.9)-(4.10). The standard errors of the MLEs $\left(\hat{\phi}_{0}, \hat{\phi}_{1}, \hat{\lambda}\right)$ are given in the third column and $95 \%$ asymptotic Wald CIs (i.e. (4.6)) of the three parameters are listed in the fourth column of Table 20. With $G=6,000$ bootstrap replications, the two $95 \%$ bootstrap CIs of $\left(\phi_{0}, \phi_{1}, \lambda\right)$ are presented in the last two columns of Table 20.

Let $\alpha=0.05$. Suppose we want to test $H_{0}:\left(\phi_{0}, \phi_{1}\right)=$ $(0,0)$ against $H_{1}:\left(\phi_{0}, \phi_{1}\right) \neq(0,0)$. According to $(5.3)$, we calculate the value of the score test statistic which is given by $t_{1}=76.6301$ and from (5.4), we have $p_{\mathrm{v} 1}=0 \ll 0.05$. Thus, the $H_{0}$ should be rejected at the significance level of $\alpha=0.05$.

Suppose we want to test $H_{0}: \phi_{1}=0$ against $H_{1}: \phi_{1}>0$. According to (5.6) and (5.9), we calculate the values of the LRT statistic and score test statistic, which are given by $t_{2}=4.4298$ and $t_{3}=5.1068$, respectively. Then, from (5.8) and (5.10), we have $p_{\mathrm{v} 2}=0.0177$ and $p_{\mathrm{v} 3}=0.0238$, both are less than 0.05 . Thus we should reject $H_{0}$.

\subsubsection{Model comparison}

The model fitting is conducted by Pearson's chi-squared test, AIC and BIC. We listed the predicted frequencies in Table 21 to compare Poisson, ZIP and ZOIP models. The computed Pearson's chi-square statistics indicate that the Poisson model exhibits a strong lack of fit and the ZIP model does not exhibit lack of fit, but the ZOIP model substantially improves the fit. Moreover, both the LRT and the score test favor the ZOIP model. However, the AIC favors the ZOIP model, while the BIC favors the ZIP model.

\section{DISCUSSION}

In this paper, we extensively study the zero-and-one inflated Poisson distribution by first establishing different but equivalent stochastic representations for the ZOIP random variable and then developing some important distributional properties. As we have seen, these stochastic representations play key roles in obtaining explicit expressions of the moments, the moment generating function, and in deriving an EM algorithm and so on. Useful results related to the conditional distributions based on the first two stochastic representations are also presented. Although the ZOIP model involves one more parameter indicating the existence of extra ones when comparing with the ZIP model, the MLEs and confidence intervals of parameters of interest
Table 21. Comparison of the fitted frequencies from Poisson, ZIP and ZOIP distribution for the ammunition factory accidents data

\begin{tabular}{l|cccc}
\hline \hline Count & $\begin{array}{c}\text { Observed } \\
\text { frequency }\end{array}$ & Poisson & ZIP & ZOIP \\
\hline 0 & 447 & 406.31 & 447.00 & 447.00 \\
1 & 132 & 189.03 & 124.60 & 132.00 \\
2 & 42 & 43.97 & 54.95 & 43.72 \\
3 & 21 & 6.82 & 16.15 & 17.48 \\
4 & 3 & 0.79 & 3.56 & 5.24 \\
5 & 2 & 0.08 & 0.73 & 1.56 \\
\hline Pearson's $\chi^{2}$ & & 103.14 & 7.22 & 1.86 \\
d.f. & & 4 & 3 & 2 \\
$p$-value & & $<0.001$ & 0.0653 & 0.3946 \\
\hline $4+$ & 5 & 0.87 & 4.30 & 6.80 \\
Pearson's $\chi^{2}$ & & 70.37 & 5.06 & 1.25 \\
d.f. & & 3 & 2 & 1 \\
$p$-value & & $<0.001$ & 0.0797 & 0.2629 \\
\hline$\phi_{0}$ & & & 0.4725 & 0.5969 \\
$\phi_{1}$ & & & & 0.0913 \\
$\lambda$ & & 0.4652 & 0.8820 & 1.1994 \\
\hline AIC & & 1236.37 & 1190.54 & $\mathbf{1 1 8 8 . 1 2}$ \\
BIC & & $\mathbf{1 1 9 9 . 4 9}$ & 1201.53 \\
\hline
\end{tabular}

can be easily obtained by the Fisher scoring algorithm or the EM algorithm, and the bootstrap methods developed in this paper. We also noted that the Pearson chi-squared goodness-of-fit test is more useful than both the AIC and BIC in model selection because the former can provide a $p$-value.

In Section 2.5, the SR (2.14) in fact defines a zero-andone adjusted (or modified, or altered) Poisson distribution that can incorporate both inflation and deflation. Therefore, it is a further extension of the ZOIP model.

In Section 4.2, the MLEs of parameters in ZOIP model were obtained through the EM algorithm specified by (4.9) and (4.10). Once the EM converged, the iteration will arrive at an equilibrium, i.e.,

$$
\begin{aligned}
\hat{\phi}_{0} & =\frac{m_{0} \hat{\phi}_{0}}{n\left[\hat{\phi}_{0}+\left(1-\hat{\phi}_{0}-\hat{\phi}_{1}\right) \mathrm{e}^{-\hat{\lambda}}\right]} \text { and } \\
\hat{\phi}_{1} & =\frac{m_{1} \hat{\phi}_{1}}{n\left[\hat{\phi}_{1}+\left(1-\hat{\phi}_{0}-\hat{\phi}_{1}\right) \hat{\lambda} \mathrm{e}^{-\hat{\lambda}}\right]} .
\end{aligned}
$$

Therefore, we obtain 
(8.1) $m_{0}=n\left[\hat{\phi}_{0}+\left(1-\hat{\phi}_{0}-\hat{\phi}_{1}\right) \mathrm{e}^{-\hat{\lambda}}\right]=n\left[\hat{\phi}_{0}+\hat{\phi}_{2} \mathrm{e}^{-\hat{\lambda}}\right]$,

(8.2) $m_{1}=n\left[\hat{\phi}_{1}+\left(1-\hat{\phi}_{0}-\hat{\phi}_{1}\right) \hat{\lambda} \mathrm{e}^{-\hat{\lambda}}\right]=n\left[\hat{\phi}_{1}+\hat{\phi}_{2} \hat{\lambda} \mathrm{e}^{-\hat{\lambda}}\right]$.

Recall that in the beginning of Section $4, m_{0}=\sum_{i=1}^{n} I\left(y_{i}=\right.$ 0 ) denotes the number of zero observations in the observed data $Y_{\mathrm{obs}}=\left\{y_{i}\right\}_{i=1}^{n}$, while $m_{1}=\sum_{i=1}^{n} I\left(y_{i}=1\right)$ denotes the number of 1 observations in $Y_{\text {obs }}$. On the other hand, from (1.1), we know that the probability of $\{Y=0\}$ is $\phi_{0}+\phi_{2} \mathrm{e}^{-\lambda}$ so that the estimated frequency of zero category should be $n\left[\hat{\phi}_{0}+\hat{\phi}_{2} \mathrm{e}^{-\hat{\lambda}}\right]$, which must be equal to $m_{0}$ according to (8.1). Similar interpretation can be applied to $m_{1}$. For example, in Table 9 , the observed frequencies for 0 and 1 are $m_{0}=134$ and $m_{1}=314$, while the fitted frequencies for 0 and 1 based on ZOIP are exactly 134 and 314. In addition, in a ZIP model (a special case of ZOIP), since we also apply the EM algorithm, it is not surprising that the fitted frequency for zero category is exactly equal to the observed frequency for the zero category.

\section{ACKNOWLEDGEMENTS}

The authors would like to thank the Editor, an Associate Editor and one referee for their helpful comments and useful suggestions.

\section{Received 27 March 2014}

\section{REFERENCES}

[1] Akaike, H. (1974). A new look at the statistical model identification. IEEE Transactions on Automatic Control 19(6) 716-723. MR0423716

[2] Böhning, D. (1998). Zero-inflated Poisson models and C.A.MAN A tutorial collection of evidence. Biometrical Journal 40(7) 833843.

[3] Carrivick, P. J. W., Lee, A. H., and Yau, K. K. W. (2003). Zero-inflated Poisson models and C.A.MAN: A tutorial collection of evidence. Safety Science 41(1) 53-63.

[4] Cohen JR., A. C. (1960a). Estimating the parameters of a modified Poisson distribution. Journal of the American Statistical Association 55 139-143. MR0111065

[5] Cohen Jr., A. C. (1960b). Estimation in the truncated Poisson distribution when zeros and some ones are missing. Journal of the American Statistical Association 55 342-348. MR0121891

[6] David, F. N. and Johnson, N. L. (1952). The truncated Poisson. Biometrics 8(4) 275-285. MR0052729

[7] Dieckmann, A. (1981). Ein einfaches stochastisches modell zur analyse von häufigkeitsverteilungen abweichenden verhaltens. Zeitschrift für Soziologie 10(3) 319-325.

[8] ERIKsson, R. and ÅberG, R. (EDs) (1987). Welfare in Transition: A Survey of Living Conditions in Sweden 1968-1981. Clarendon Press, Oxford.

[9] Feng, Z. D. and McCulloch, C. E. (1992). Statistical inference using maximum likelihood estimation and the generalized likelihood ratio when the true parameter is on the boundary of the parameter space. Statistics \& Probability Letters 13(4) 325-332. MR1160755

[10] Greenwood, M. and Yule, G. U. (1920). An inquiry into the nature of frequency distributions representative of multiple happenings with particular reference to the Occurrence of multiple attacks of disease or of repeated accidents. Journal of the Royal Statistical Society 83(2) 255-279.

[11] Gupta, P. L., Gupta, R. C., and Tripathi, R. C. (1996). Analysis of zero-adjusted count data. Computational Statistics \& Data Analysis 23(2) 207-218.

[12] Hasselblad, V. (1969). Estimation of finite mixtures of distributions from the exponential family. Journal of the American Statistical Association 64 1459-1471.

[13] Hilbe, J. M. (2011). Negative Binomial Regression, 2nd ed. Cambridge University Press, New York. MR2797563

[14] Jansakul, N. and Hinde, J. P. (2002). Score tests for zeroinflated Poisson models. Computational Statistics \& Data Analysis 40 75-96. MR1921123

[15] Joe, H. and Zhu, R. (2005). Generalized Poisson distribution: The property of mixture of Poisson and comparison with negative binomial distribution. Biometrical Journal 47(2) 219-229. MR2137236

[16] Kemp, A. W. (1986). Weighted discrepancies and maximum likelihood estimation for discrete distributions. Communications in Statistics - Theory and Methods 15(3) 783-803. MR0832079

[17] LAmBerT, D. (1992). Zero-inflated Poisson regression, with an application to defects in manufacturing. Technometrics 34(1) 114.

[18] Leroux, B. G. and Puterman, M. L. (1992). Maximumpenalized-likelihood estimation for independent and Markovdependent mixture models. Biometrics 48 545-558.

[19] Martin, D. C. and Katti, S. K. (1965). Fitting of certain contagious distributions to some available data by the maximum likelihood method. Biometrics 21(1) 34-48. MR0182101

[20] Melkersson, M. and Olsson, C. (1999). Is visiting the dentist a good habit? Analyzing count data with excess zeros and excess ones. Umeå Economic Studies, No. 492, Umeå University, Umeå, Sweden.

[21] Mullahy, J. (1986). Specification and testing of some modified count data models. Journal of Econometrics 33(3) 341-365. MR0867980

[22] Neyman, J. (1939). On a new class of "contagious" distributions, applicable in entomology and bacteriology. The Annals of Mathematical Statistics 10(1) 35-57.

[23] Pearson, K. (1900). On the criterion that a given system of deviations from the probable in the case of a correlated system of variables is such that it can be reasonably supposed to have arisen from random sampling. Philosophical Magazine Series 5 50(302) $157-175$.

[24] Ridout, M., Demétrio, C. G. B., and Hinde, J. (1998). Models for count data with many zeros. Invited Paper in the XIX-th International Biometric Conference, Cape Town.

[25] Saito, M. Y. and Rodrigues, J. (2005). A Bayesian analysis of zero and one inflated distributions. Rev. Mat. Estat. 23(1) 47-57. MR2304490

[26] Schilling, W. (1947). A frequency distribution represented as the sum of two Poisson distributions. Journal of the American Statistical Association 42 407-424. MR0021280

[27] Schwarz, G. (1978). Estimating the dimension of a model. The Annals of Statistics 6(2) 461-464. MR0468014

[28] Self, S. G. and Liang, K. Y. (1987). Asymptotic properties of the maximum likelihood estimators and likelihood ratio tests under nonstandard conditions. Journal of the American Statistical Association 82 605-610. MR0898365

[29] Singh, S. N. (1963). A note on inflated Poisson distribution. Journal of the Indian Statistical Association 1(3) 140-144. MR0162309

[30] Tanner, M. A. and Wong, W. H. (1987). The calculation of posterior distributions by data augmentation. Journal of the American Statistical Association 82(398) 528-540. MR0898357 
Chi Zhang

Department of Statistics and Actuarial Science

The University of Hong Kong

Pokfulam Road

Hong Kong

P.R. China

E-mail address: zhangchi@hku.hk

Guo-Liang Tian

Department of Statistics and Actuarial Science

The University of Hong Kong

Pokfulam Road

Hong Kong

P.R. China

E-mail address: gltian@hku.hk
Kai-Wang Ng

Department of Statistics and Actuarial Science

The University of Hong Kong

Pokfulam Road

Hong Kong

P.R. China

E-mail address: kaing@hku.hk 